\title{
LA DOCTRINA NETANYAHU. UN CAMBIO DE PARADIGMA EN LA POLÍTICA EXTERIOR DEL ESTADO DE ISRAEL
}

\author{
Alberto Priego ${ }^{1}$ \\ Universidad Pontificia de Comillas
}

Resumen:

En los casi 75 años de existencia del Estado de Israel, su política exterior ha mantenido una cierta continuidad. Algunos primeros ministros como David Ben-Gurión, Menahem Begin, Issac Rabín han dado giros a la política exterior que, a largo plazo, han condicionado el futuro de Israel. El pasado verano Benjamín Netanyahu salió del gobierno después de más de doce años en el cargo convirtiéndose en el mandatario israelí que más tiempo ha permanecido en el cargo. A lo largo de estos años, Benjamín Netanyahu ha introducido importantes reformas estructurales en país. Uno de estos campos ha sido la política exterior donde se puede considerar que se ha instalado una doctrina propia, la Doctrina Netanyahu. Este artículo tratará de construir los puntos fundamentales que componen esta doctrina de política exterior. Se adoptará una aproximación interpretativa usando los discursos más importantes de Benjamín Netanyahu.

Palabras Clave: Israel, Netanyahu, Irán, Proceso de Paz, Economía, Política Exterior.

Title in English: The Netanyahou Doctrine: A paradigm shift in the State of Israel's foreign policy

\section{Abstract:}

After almost 75 years of existence of the State of Israel, its foreign policy has maintained a certain continuity. Some prime ministers such as David Ben Gurion, Menahem Begin, Isaac Rabin have taken some turns in foreign policy that in the long term will condition Israel's future. Last summer Benjamin Netanyahu left the government after more than twelve years in office, becoming the longest-serving Israeli leader. Throughout these years, Benjamin Netanyahu has introduced important structural reforms in the country. One of these fields has been foreign policy, where he has implemented his own doctrine, the Netanyahu Doctrine. This article will try to present the fundamental points that make up this foreign policy doctrine. An interpretative approach will be adopted using the most important speeches of Benjamin Netanyahu.

Key words: Netanyahu, Iran, Peace Process, Economy, Foreign Policy.

Copyright (C) UNISCI, 2021.

Las opiniones expresadas en estos artículos son propias de sus autores, y no reflejan necesariamente la opinión de UNISCI. The views expressed in these articles are those of the authors, and do not necessarily reflect the views of UNISCI.

\footnotetext{
${ }^{1}$ Alberto Priego es Profesor Propio Agregado del Departamento de Relaciones Internacionales de la Universidad Pontificia de Comillas

E-mail: <apriego@icade.comillas.edu>

DOI: http://dx.doi.org/10.31439/UNISCI-125
} 


\section{Introducción}

Desde el año 1945, hemos asistido a la emergencia de una suerte de esfuerzos teóricos para sistematizar las diferentes políticas exteriores de los Estados, sobre todo en regímenes presidenciales. Casi todos los presidentes norteamericanos han sido objeto de estos estudios, por lo que a todos nos resultan más o menos familiares conceptos tales como Doctrina Bush ${ }^{2}$, Doctrina Reagan ${ }^{3}$ o Doctrina Truman ${ }^{4}$. En el caso de la Unión Soviética -después Rusiatambién encontramos una gran cantidad de trabajos sobre su política exterior generando conceptos tan conocidos como la Doctrina Brezhnev ${ }^{5}$, Primakov, Gerasimov ${ }^{6}$ o incluso algunos se atreven a escribir sobre la vigente Doctrina Putin ${ }^{7}$

Si nos atenemos a estos trabajos, se podría pensar que el concepto "doctrina" está reservado a las políticas exteriores de las superpotencias o cuanto menos a la dimensión exterior de los regímenes presidencialistas. Sin embargo, nada más lejos de la realidad ya que en los regímenes parlamentarios también encontramos estudios sobre diferentes doctrinas en política exterior como la Doctrina Thatcher ${ }^{8}$ o la Blair ${ }^{9}$ Incluso hemos visto como algunos ministros de exteriores como Walter Hallstein ${ }^{10}$ o Ahmet Davotoglu ${ }^{11}$ han dado nombre a modelos de estudio de política exterior. Por lo tanto, no podemos afirmar que el concepto doctrina sea algo exclusivo ni de las superpotencias ni los regímenes presidenciales.

El caso de Israel tampoco es una excepción ya que encontramos dos primeros ministros -David Ben-Gurión ${ }^{12}$ y Menahem Begin- que han dado nombre a diferentes doctrinas de política exterior y de seguridad. Mientras que el primero marcó una forma de relacionarse con los vecinos -Doctrina de la Periferia ${ }^{13}$ - el segundo estableció el ataque preventivo ${ }^{14}$, como forma de defensa cuando Israel percibe que su supervivencia podría estar en juego.

2 García, David: "Peace Through Primacy. La Administración Bush, La política exterior de EE.UU y las bases de una primacía Imperial. Geopolítica, recursos energéticos y Guerra al Terrorismo" Unisci Discussion Papers, vol. $\mathrm{n}^{\mathrm{o}} 1$ (January 2004), pp. 1-31.

3 Scott, James M. (1996): Deciding to Intervene: The Reagan Doctrine and American Foreign Policy. Durham, Duke University Press; Lagon, Mark (1994): The Reagan Doctrine: Sources of American Conduct in the Cold War's Last Chapter. Westport, Praeger.

4 Bostdorff, Denise (2008): Proclaiming the Truman Doctrine: The Cold War Call to Arms. Austin, Texas A \& M University Press

5 También conocida como Doctrina de Soberanía Limitada. Glazer, Stephen “The Brezhnev Doctrine”, The International Lawyer, vol. 5, no 1 (January, 1971), pp. 169-179; Ouimet, Matthew (2004) The Rise and Fall of the Brezhnev Doctrine in Soviet Foreign Policy. Chapel Hill, University of North Carolina Press.

6 Rumer, Eugene (2019) The Primakov (Not Gerasimov) Doctrine in Action. Washington D.C, The Carnegie Endowment for Peace.

7 Aron, Leon: “The Putin Doctrine. Russia's Quest to Rebuild the Soviet State”, Foreign Affairs, March 2013.

8 Elles, Diane: "The Foreign Policy of the Thatcher Government" en Minogue, Kenneth and Biddiss, Michael (eds) (1987): Thatcherism: Personality and Politics, Zurich, Springer.

9 Atkins, Judi “A New Approach to Humanitarian Intervention? Tony Blair's "Doctrine of the International Community" British Politics, vol. 2, no 1 (February 2006), pp. 274-283

10 Gray, William (2003): Germany's Cold War: The Global Campaign to Isolate East Germany, 1949-1969, Chapel Hill, University of North Carolina Press

11 Stein, Aaron (2016): Turkey's New Foreign Policy: Davutoglu, the AKP and the Pursuit of Regional Order. Abingdon, Routledge.

12 En 1953 David Ben-Gurión presentó a su gabinete una doctrina de seguridad que es la que ha estado en vigor hasta hoy.

13 Mediante la Doctrina de la Periferia, Israel establecía alianzas con minorías étnicas y religiosas residentes en estados árabes vecinos (Kurdos o Maronitas), con estados no árabes y/o no musulmanes, y sobre todo con estados musulmanes no árabes (Irán o Turquía).

14 El 7 de junio de 1981 Israel bombardeó el reactor nuclear iraquí de Osirak ante la amenaza de que el Irak de Sadam Hussein pudiera desarrollar armas nucleares. Posteriormente, el 6 de septiembre de 2007 Israel bombardeaba el reactor nuclear de Al-Kibar. 
A pesar de su reciente salida del gobierno, Benjamín Netanyahu se ha convertido en el primer ministro israelí que más tiempo ha estado al frente del país, incluso por delante de David BenGurión. Por ello, cabe plantearse si en los años que estuvo al frente del ejecutivo israelí, Bibi como se le conoce en Israel, tuvo la voluntad de establecer un modelo de política exterior y de seguridad que en el futuro pueda estudiarse como "Doctrina Netanyahu".

\section{Estado de la Cuestión.}

Desde su aparición en los 90, la figura de Benjamín Netanyahu ha suscitado un gran interés en la comunidad académica. Algunos autores como Ben Caspit ${ }^{15}$, Neil Lochery ${ }^{16}$, Anshel Pfeffer ${ }^{17}$ o Hoffman han dedicado monográficos a la figura del primer ministro israelí tanto en el plano público como en el plano privado. Otros autores como Guy Ziv ${ }^{18}$ o Aluf Benn ${ }^{19}$ han preferido centrarse en un asunto o en una política concreta elaborando trabajos menos generales y más específicos. Siguiendo esta segunda línea, académicos como Raffaella del $\mathrm{Sarto}^{20}$, Doron Navot o Aviad Rubin ${ }^{21}$ han centrado sus trabajos en el liderazgo personal de Netanyahu y sobre todo, en su capacidad para ganar elecciones cuando todo parecía estar en su contra. También tenemos que mencionar a aquellos como Steven Bayme ${ }^{22}$ o Jonathan Broader ${ }^{23}$ que han focalizado su interés en la siempre complicada relación de Netanyahu con la diáspora, especialmente con los judíos que residen en los Estados Unidos. Precisamente esta relación se ha forjado a través de los viajes anuales que el primer ministro realizó a los Estados Unidos, momento en el que Netanyahu aprovechaba para realizar discursos ante diferentes auditorios (Congreso de los Estados Unidos, el AIPAC o la Asamblea General de las Naciones Unidas). Por este motivo autores como Michal Ephratt ${ }^{24}$, Justin Scott Finkelstein ${ }^{25}$ o Bernard Avisai ${ }^{26}$ han estudiado esta distintiva faceta de Benjamín Netanyahu.

A pesar de ser conocido por su faceta puramente política, entre 2003 y 2005 Netanyahu ostentó el cargo de ministro de finanzas en el gabinete de Ariel Sharon. Por ello, hay un importante grupo de autores entre los que destacan Igal Charney ${ }^{27,}$ Dany Bahar ${ }^{28,}$ Zvi Eckstein ${ }^{29}$ o Shlomo

15 Caspit, Ben (2016): The Netanyahu Years, London, Thomas Dunne Books.

16 Lochery, Neil (2016): The Resistible Rise of Benjamin Netanyahu. London, Bloomsbury.

17 Pfeffer, Anshel (2017): Bibi: The Turbulent Life and Times of Benjamin Netanyahu. London, Basic Civitas Books.

18 Ziv, Guy: "Benjamin Netanyahu's Calculated Ambiguity Toward the Two-State Solution"

Political Science Quarterly, vol. 13, no 2 (2018-19).

19 Benn, Aluf: "The End of the Old Israel: How Netanyahu has transformed the nation" Foreign Affairs, vol. 96, no 4 (2016) pp.16-27.

20 del Sarto, Raffaella "Back to Square One? The Netanyahu Government and the Prospects for Middle East Peace", Mediterranean Politics, vol. 14, n 3 (November 2009), pp. 421-428.

21 Navot, Doron and Rubin, Aviad: "Likud's success in the 2015 elections: Netanyahu's Hobbesian moment" Israel Affairs, vol. 22, no 3-4, pp. 628-640.

22 Bayme, Steven "Israel-diaspora relations in the age of Netanyahu” en Freedman, Robert O. (2019): Israel Under Netanyahu: Domestic Politics and Foreign Policy, Abingdon, Routledge.

23 Broader, Jonathan "Netanyahu and the American Jew" World Policy Journal, (Spring 1998), pp. 89-98.

24 Ephratt, Michal "The Silence Address: Silence as it Emerged from Media Commentators and Respondents, Following Prime Minister Netanyahu's 2015 Address at the UN” Israel Studies, vo. 22, no 3 (Fall 2017).

25 Finkelstein, Justin S. "Netanyahu's Speech: What Difference Does it Make?" Foreign Policy Research Institute, (March 2015).

26 Avisai, Bernard: "Netanyahu's Speech" New Yorker" (March 2015) en

https://www.newyorker.com/news/news-desk/netanyahu-speech-congress

27 Charney, Igal "A "Supertanker" Against Bureaucracy in the Wake of a Housing Crisis: Neoliberalizing Planning in Netanyahu's Israel” Antipode, vol. 49, no 5 (2017), pp. 1223-1243

28 Bahar, Dany (2016): "Delivering on economic prosperity in Israel. How monopolies are hampering the start up nation", Brookings Institute (4.5.2016)

29 Bahar, Dany and Eckstein, Zvi "Israeli Voters Don't Care About the Economy. They Should" Foreign Policy, (September 2019) 
Svirsky ${ }^{30}$ que han centrados sus investigaciones en el exitoso, pero a la vez controvertido modelo económico de Netanyahu.

El autor que más y mejor ha tratado la relación de Israel con Estados Unidos ${ }^{31}$ es sin lugar a duda Robert O. Freedman. Además de ser el autor de innumerables trabajos sobre la relación entre Jerusalén y Washington, Freedman ha coordinado un volumen que se centra precisamente en los años en los que Netanyahu ha sido primer ministro ${ }^{32}$. Este trabajo multidisciplinar de reciente publicación analiza entre otros asuntos su política exterior, su relación con los palestinos o su relación con los partidos religiosos. Se trata sin duda de la obra académica más completa sobre los años de Netanyahu en el gobierno.

El interés por Netanyahu y por sus políticas, no se agota en la academia israelí o en la norteamericana. También los académicos palestinos, o los autores no palestinos pero cercanos a posiciones palestinas, han mostrado interés por la figura del primer ministro, aunque evidentemente sus textos tienen un cariz distinto. Entre otros muchos podemos destacar los trabajos de Shaqued Morag ${ }^{33}$, de Daoud Kuttab ${ }^{34}$ o de Laura Draker ${ }^{35}$ creadora esta última del concepto "primer" para referirse a Netanyahu.

\section{En España no son muchos los autores que han tratado esta cuestión. ${ }^{36}$}

A pesar de este interés por Netanyahu, no encontramos estudios que traten de sistematizar su política exterior. Por ello, este artículo buscará ver en qué consiste la dimensión exterior de sus años de gobierno y en qué medida este legado puede marcar una línea similar a la que en su día marcó David Ben-Gurión.

\section{Preguntas y objetivos de Investigación}

Tal y como hemos destacado en el apartado anterior, podemos afirmar que existen muchos y muy buenos trabajos que tratan de analizar tanto la persona de Netanyahu como las políticas que se han desarrollado durante su mandato. Sin embargo, después de haber estudiado estas obras, cabe plantearse algunas cuestiones, que serán las preguntas de investigación de este artículo.

1. ¿La política exterior de Netanyahu introduce elementos rupturistas en la política exterior de Israel o en cambio mantiene la línea de sus predecesores?

2. ¿Qué tiene de nuevo y qué elementos mantiene de sus predecesores?

30 Svirsky, Shlomo (2013): "Nes Gadol Haya Po. The economic policy of the second Netanyahu government, 2009-20122, Adva Center (Noviembre 2013)

31 Freedman, Robert O. "The erosion of US-Israeli relations during Obama's second term", Israel Affairs, n 2 (23), pp. 253-272.

32 Freedman, Robert O. (2019): Israel Under Netanyahu: Domestic Politics and Foreign Policy. Abingdon, Routledge.

33 Morag, Shaqued "From Rabin's Peace to Netanyahu's Peace Conflict Management: The Ethos of and Israel's Younger Generation" Palestine Israel Journal, vol. 26, n 1-2, (2019), pp. 42-49.

34 Kuttab, Daoud "Executive Overreach and Its Disastrous Results: The Case of Trump and Netanyahu" Palestine Israel Journal, nº 3-4 (24) (2017), pp. 175-180.

35 Drake, Paula "Netanyahu Primer", Journal of Palestine Studies, 1996, vol. 26, no 1 (Autumn, 1996), pp. 58-69.

${ }^{36}$ Tenemos que destacar algunos trabajos publicados en la Editorial Política Exterior como los de Julio de la Guardia, Alain Dieckhoff, Amos Harel, Jaime Ojeda o Ignacio Álvarez Osorio. En el campo periodístico encontramos bastantes contribuciones como las de Alfonso Palomares, Miguel Ángel Benedicto o a Ximena Marín. En el ámbito de los think tank, tenemos que destacar los trabajos publicados en el Instituto Español de Estudios Estratégicos donde encontramos las aportaciones de Fernando Prieto Arellano y José Ignacio Torres. Sin embargo, aún estamos lejos de otros países donde el tema de Israel en general y el de Netanyahu en particular ha generado una mayor producción intelectual. En el campo periodístico encontramos contribuciones de Alfonso Palomares, Miguel Ángel Benedicto o a Ximena Marín. 
3. ¿En qué aspectos se basa?, ¿en qué puntos hace más incidencia?, ¿cuáles han sido sus prioridades?

4. ¿En qué pilares se basará? ¿en la diplomacia, en la economía o en los aspectos relacionados con la seguridad?

5. ¿Puede considerarse como una política exterior que perdurará en el tiempo?

Estas preguntas deberán servirnos para responder a muchas cuestiones cuya respuesta no ha sido posible encontrarla en las lecturas que hemos revisado en el "Estado de la Cuestión". Así, podemos establecer como objetivo fundamental del artículo aclarar si existe, o no, una Doctrina Netanyahu y en el caso de que exista ver en qué consiste y qué implicaciones puede tener para Israel y para la región de Oriente Medio.

\section{Metodología}

El presente artículo va a adoptar un enfoque interpretativo, usando para ello técnicas de investigación cualitativas que nos ayudarán tanto a responder a las preguntas de investigación, como a alcanzar los objetivos planteados. En todo trabajo las fuentes analizadas poseen una gran importancia, pero en este ésta será un mayor. Vamos a intentar explicar esta cuestión. En este artículo hemos utilizado tanto fuentes primarias como fuentes secundarias.

En lo que a las fuentes primarias se refiere, usaremos un total de 18 discursos (fuentes primarias archivísticas ${ }^{37}$ ) pronunciados por Benjamín Netanyahu a lo largo de su segunda etapa como primer ministro (2009-2021). La elección de los discursos se ha basado siguiendo un criterio material, ya que todos tienen por tema central la dimensión exterior. Estos son los discursos elegidos conforme al año y al lugar donde se pronunciaron:

- Universidad Bar-Ilán en 2009 (Tel Aviv).

- Asamblea General de las Naciones Unidas en 2011, 2012, 2013, 2014, 2015, 2016, 2017, 2018, 2020 (Nueva York).

- Congreso de los Estados Unidos 2011 y 2015.

- AIPAC 2012, 2013, 2014 y 2018 (Washington).

- Knesset 2021(Jerusalén)

- La Casa Blanca 2020 (Washington).

Para el análisis de estos discursos, hemos contado con la ayuda del programa de investigación cualitativa Nvivo12, con el que hemos trabajado en estos 18 documentos con el fin de establecer si existe o no una Doctrina Netanyahu. Para ello, se han seleccionado un total de 71 conceptos $^{38}$ (Ver ANEXO 2) presentes en los 18 discursos. Así, se han analizado aspectos tales como la frecuencia en la que aparecen los conceptos, su importancia en los discursos anteriormente destacados o el significado que los actores dan a los mismos.

\footnotetext{
37 Se han usado los siguientes archivos. The Jewish Virtual Library, The Times of Israel, Ministerio de Asuntos Exteriores de Israel, The Washington Post, Haaretz y Likud.

38 1948, 1967,Abbas, Al Aqsa, Al Fatah, Allah, American Embassy, Arab, Arafat, Bahrain company, conflict, diplomacy, diplomatic, economic, economy, Egypt, embassy, energy, entrepreneurship, Future, Hamas, Hezbollah, Homeland, Hope International Community, investment, Iran, Iranian Threat, Iraq, Islam, Islamic, Islamic Terrorism, Israel, Jerusalem, Jewish People, Jordan, Jordan Valley, Judea, Khomeini, Knesset, Land, Missile, money, Nuclear, Nuclear Weapons, Oslo, Palestinian, Palestinian Authority, Palestinian State, Peace, Peace Process, Prosperity, Reconciliation, Refugees, Samaria, Saudi Arabia, Security, start up, Syria, technology, Tehran, Temple Mount, Terrorism, Terrorist, Threat, trade, two states, UAE, United States, War, Western Wall.
} 
En lo que a las fuentes secundarias se refiere, se usarán fuentes bibliográficas, hemerográficasdivulgativas $^{39}$, hemerográficas-científicas ${ }^{40}$, técnicas ${ }^{41}$ e incluso audiovisuales.

Para estructurar el artículo, hemos partido del discurso pronunciado en 2009 en la Universidad de Bar-Ilan por tratarse del primero y ser una declaración de intenciones de lo que iba a hacer en sus años de gobierno. De este discurso hemos recogido los tres aspectos que Netanyahu destaca como desafíos de su política exterior ${ }^{42}$ y que servirán para construir su modelo o doctrina.

- La amenaza iraní

- La crisis financiera

- El proceso de paz.

Estos desafíos serán la base sobre la que se creará el modelo o Doctrina Netanyahu. Así, tratando de frenar la proliferación iraní, Netanyahu ha creado un modelo propio de seguridad basado en alianzas con las monarquías sunitas que aísla a Irán de su contexto regional. Por otro lado, buscando superar la crisis Netanyahu ha transformado y modernizado la economía israelí, y por último a través del maltrecho proceso de paz Netanyahu ha cambiado la lógica de la negociación pasando de paz por territorios a paz por prosperidad.

El artículo se va a estructurar de la siguiente forma. En primer lugar, se van a destacar los aspectos generales de la política exterior de Netanyahu, para pasar a continuación a analizar cada uno de los tres grandes desafíos: a) Irán, b) la economía y c) el proceso de paz. Sobre estos tres desafíos veremos como se han configurado los tres grandes pilares de su doctrina: a) pilar de la seguridad, b) pilar de la economía y c) pilar de la diplomacia.

\section{La Doctrina Netanyahu}

Lo que tratamos de demostrar es que durante los últimos 12 años en los que Benjamín Netanyahu ha estado al frente del Estado de Israel, se ha consolidado un modelo de política exterior propio que sobrevivirá a su propio mandato. Partiendo de las tres prioridades establecidas en el discurso de Bar-Ilán -Irán, Crisis Financiera y Proceso de Paz- Netanyahu ha establecido un modelo de política exterior propio basado en:

- Pilar Seguridad: Partiendo de la amenaza iraní se busca la eliminación preventiva de amenazas apocalípticas basadas en el desarrollo de armas de destrucción masiva por parte de sus vecinos.

- Pilar Económico: Partiendo de la crisis financiera se busca la transformación del modelo económico israelí creando uno propio basado en la tecnología y en la exportación de energía.

\footnotetext{
39 Entendemos por fuente hemerográfica-divulgativa aquella que mantiene un carácter periódico pero que carece de doble ciego. En este trabajo se han usado las siguientes: Foreign Affairs, New Yorker, The Economist y World Policy Journal.

40 Entendemos por fuente hemerográfica-científica aquella de carácter periódico que está sometida al doble ciego. En este trabajo se han usado las siguientes: British Politics, Foreign Affairs, Israel Affairs, Israel Studies, Journal of Palestine Studies, Mediterranean Politics, Palestine Israel Journal, Presidential Studies Quarterly, Political Science Quaterly, The International Lawyer, Unisci Discussion Papers.

41 Entendemos por fuentes técnicas (papers) los documentos producidos por think tanks que sin periodicidad establecida ni requerimientos científicos pretenden recoger y analizar información. En este trabajo se han usado los siguientes: Brookings Institute, Avda Center, UNISCI, Real Instituto Elcano, FAES e Instituto Español de Estudios Estratégicos.

42 "We are currently facing three tremendous challenges: The Iranian threat, the financial crisis, and the promotion of peace" Netanyahu, Benjamin (2009): Israeli PM Netanyahu's Bar-Ilan Speech (2009), en https://ecf.org.il/media_items/1141,
} 
- Pilar Diplomático: Partiendo de los planes fracasados de negociación se busca la superación del conflicto cambiando la lógica de "paz por territorios" por la de "paz por prosperidad".

Estos tres pilares están íntimamente relacionados entre sí, ya que no son elementos complementarios sino completivos. En otras palabras, la existencia de la Doctrina Netanyahu solo es posible si existen al mismo tiempo los tres pilares ya que, si uno fallara, toda la doctrina se vendría abajo.

Gráfico 1: Los pilares de la Doctrina Netanyahu.

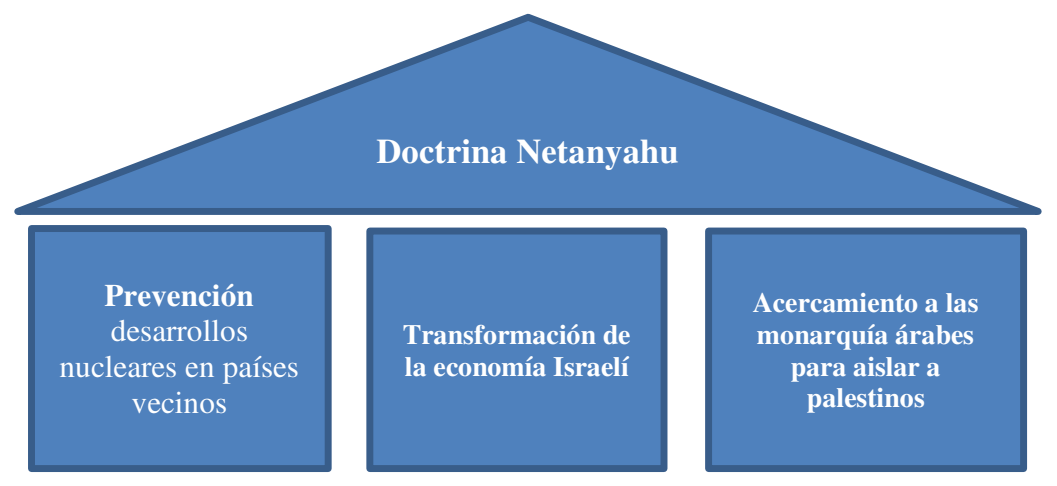

Fuente: Elaboración propia

Como se aprecia en el gráfico 2, la relación entre los tres pilares se refiere, hay que decir que éstos no ocupan posiciones equiparables. Si analizamos las alusiones que Netanyahu hace a las palabras diplomacia (8 veces), economía (27 veces) y seguridad (102 veces) en los 18 discursos seleccionados, podemos afirmar que al igual que en los modelos anteriores, en la Doctrina Netanyahu prima por encima de los otros dos la seguridad ${ }^{43}$.

Gráfico 2: Equilibrio entre los tres pilares (Seguridad, Economía y Diplomacia)

\section{Relación entre Seguridad, Economía y Diplomacia}



Fuente: Elaboración propia

Sin embargo, hay un esfuerzo explícito por desecuritizar o al menos ocultar este aspecto. ¿Por qué? La respuesta la tenemos que encontrar en la necesidad de acercarse a las monarquías árabes y en la búsqueda de inversión y comercio. Estos hechos han provocado que Netanyahu haga un

43 Snajder, Mario "Seguridad en Israel: un concepto flexible y cambiante" en Leal, Inma L., y Moloeznik, Marcos P. (Coord.) (2017): La política de defensa y seguridad de Israel como modelo, Guadalajara, Universidad de Guadalajara. 
esfuerzo, ya sea real o ficticio, por eliminar o cuanto menos maquillar ${ }^{44}$ los aspectos de la seguridad de su política exterior.

La prueba de esta des-securitización la encontramos en el gráfico 3 donde apreciamos como a lo largo de los años de gobierno, Netanyahu reduce en sus discursos las alusiones directas a los conceptos relacionados de forma directa con la seguridad. Así, vemos que conceptos como conflict, terrorism, islamic terrorism, terrorist, missile y threat), se hacen menos frecuentes a partir de 2017, llegando incluso a convertirse en irrelevantes.

Gráfico 3: Términos relacionados con la Seguridad



Fuente: Elaboración propia

Otro elemento muy significativo, es el uso repetido de términos nacionalistas, algo que se hace especialmente intenso en los momentos de crisis. En los discursos analizados, encontramos no solo un uso intenso de los conceptos Israel (918 veces) y Jewish People (405), sino que como hemos dicho anteriormente estos conceptos aparecen con más frecuencia en los momentos de mayor tensión con sus vecinos. Como se aprecia en el gráfico 4, los momentos en los que Netanyahu recurrió con mayor asiduidad a términos de carácter nacionalista fue en los periodos 2011-2012, 2014-2016 y en el año 2018. Veamos que hechos ocurrieron durante estos años para provocar que Netanyahu tuviera que acudir a ellos.

- Periodo $2011^{45}$ y 2012 ${ }^{46}$. En el año 2011, Israel sufrió una serie de ataques terroristas en el sur del país a manos del Comité de Resistencia Popular dejando un total de 8 muertos y más de 40 heridos. Un año más tarde, Israel lanzó la Operación Pilar Defensivo contra Hamás en la Franja de Gaza. En lo que a Irán se refiere, entre los años 2011 y 2012 dos científicos iraníes vinculados al programa nuclear (Darius Rezainejad y Mostafá Ahmadi Roshen) fueron asesinados en Irán. Además, Irán sufrió una serie de sabotajes en las instalaciones nucleares situadas en Isfahán y Bidganeh ${ }^{47}$. Todos estos hechos coincidieron con una intensificación de las alusiones nacionalistas en los discursos de Netanyahu

- Periodo 2014 y 2016 ${ }^{48}$. Debido a la firma del acuerdo nuclear con Irán, Israel intensificó la presión internacional sobre el país persa. En agosto de 2014 un drone israelí fue

\footnotetext{
${ }^{44}$ Para ello, se ha usado diferentes herramientas vinculadas en su mayor parte a la Hasbará (esclarecimiento) o Diplomacia Pública.

45 En el año 2011 Israel sufrió una serie de atentados en el sur.

46 En el año 2012 Israel llevó a cabo la Operación Pilar defensivo contra Hamás en Gaza

47 Priego, Alberto (2021): "La inaplicabilidad de la Doctrina Begin en Irán: la Doctrina Bar Kojba” Documentos de Opinión IEEE, 70/2021, p. 19, en

http://www.ieee.es/Galerias/fichero/docs_opinion/2021/DIEEEO70_2021_ALBPRI_Iran.pdf

48 En julio de 2014 Israel llevó a cabo la Operación Margen Protector contra Hamás en Gaza. Entre 2014 y 2016
} 
derribado mientras sobrevolaba la central de enriquecimiento de uranio de $\operatorname{Natanz}^{49} \mathrm{y}$ en octubre se produjo un sabotaje en las instalaciones de desarrollo de misiles de Parchin $^{50 .}$ También en este año, entre julio y agosto de 2014, Israel lanzó una ofensiva sobre Gaza denominada Operación Margen Protector. De nuevo, al tiempo que ocurrían estos hechos, Netanyahu incrementó el uso de términos nacionalistas en sus discursos.

- Año 2018. Tras la publicación del proyecto AMAD, Israel intensificó la presión internacional sobre Irán. Como respuesta a este movimiento el líder de la Fuerza Quod, el General Suleimani, amenazó a Israel con un ataque ${ }^{51}$ que le borrara del mapa. La amenaza vino acompañada de una ofensiva con misiles Qasam contra Israel, lo que provocó un ataque contra las bases iraníes en Siria en los Altos del Golán. Además, el traslado de la Embajada de los Estados Unidos a Jerusalén, provocó graves disturbios provocados por los palestinos. Estos hechos explican porque en el año 2018 Netanyahu recurrió de forma más frecuente a los términos nacionalistas.

Gráfico 4 Alusiones a Israel y al pueblo judío en los discursos de Netanyahu

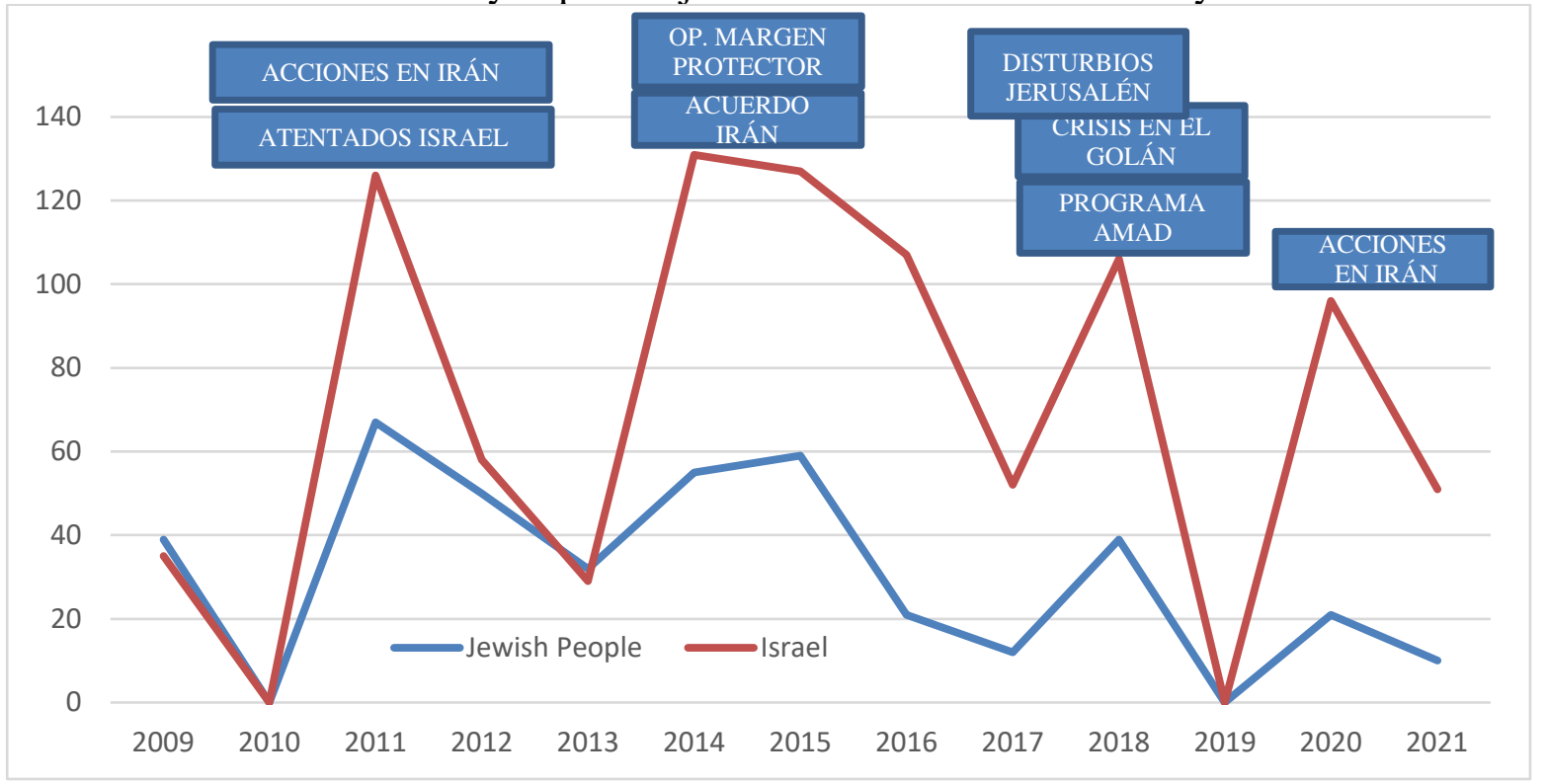

Fuente: Elaboración propia

Si nos centramos en algunos términos más concretos como Jerusalem, Samaria, Judea, Western Wall, Land, Temple Mount, 1948, 1967 o Jordan Valley, apreciamos una coincidencia absoluta entre las crisis y los momentos en los que Netanyahu los utiliza con más frecuencia en sus discursos.

Israel llevó a cabo operaciones de sabotaje contra Irán para retrasar el programa nuclear.

49 Espinosa, Ángeles: "Irán dice que ha derribado un dron israelí cerca de una planta nuclear" El País, 24 de agosto de 2014, en

https://elpais.com/internacional/2014/08/24/actualidad/1408890821_065821

50 Marcus, Jonathan "'Blast' deepens mystery of Iran's Parchin military complex" $B B C, 9$ October 2021, en https://www.bbc.com/news/world-middle-east-29550156

51 Staff, Toi: "Head of Iran's secret operations overseas threatens to 'erase Zionist entity"”, Times of Israel, 15 February 2018. 
Gráfico 5: Alusiones nacionalistas en el discurso de Netanyahu.

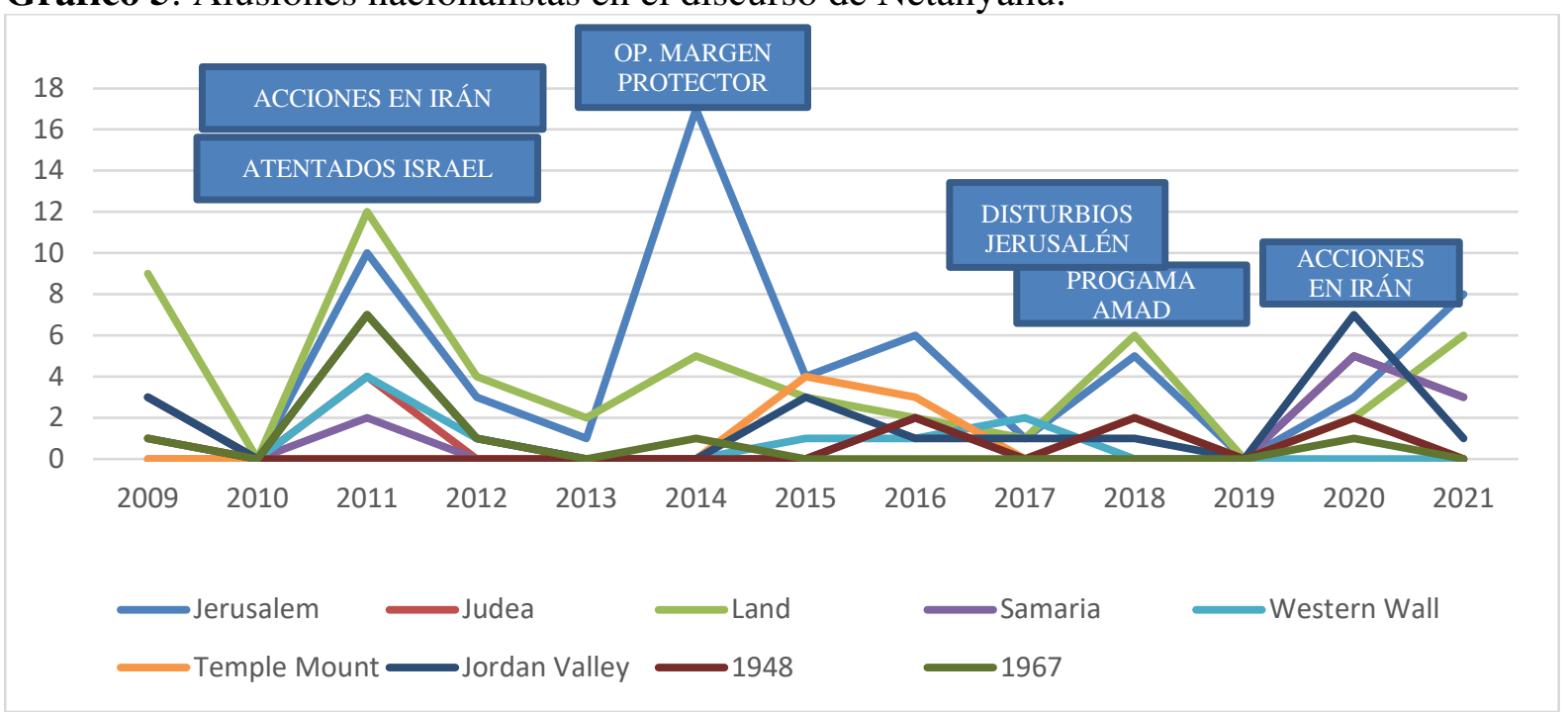

Fuente: Elaboración propia

El recurso del nacionalismo como elemento legitimador de la gestión de las crisis, no es ni mucho menos nuevo. Otros partidarios del sionismo revisionista como Menachem Begin, Ariel Sharon o Elhud Olmert ya acudieron a este recurso con anterioridad. Por lot tanto, en este punto encontramos una cierta continuidad con otros líderes que precedieron a Netanyahu en su posición.

Una vez analizados los aspectos más generales, vamos a entrar a analizar los diferentes pilares de la doctrina. Partiremos de la prioridad destacada por Netanyahu en su discurso de Bar Ilán para ir construyendo posteriormente cada uno de los tres pilares.

\subsection{Pilar de la Seguridad (Amenaza Iraní)}

Irán es desde hace ya algunos años el principal problema de seguridad de Israel. Son muchos los primeros ministros -Sharon, Olmert etc...- que han identificado a Irán como la fuente que puede amenazar a Jerusalén. Sin embargo, la relación de Netanyahu con Irán no es ni mucho menos sencilla. Cuando se convirtió en primer ministro por primera vez en el año 1996, su posición respecto del país persa era muy distinta de la que adoptó en 2009. En 1996, Netanyahu -coincidiendo con la opinión del Mossad- consideraba que Irán no era la principal amenaza de Israel. En el año 1996 Netanyahu apostó por poner toda la presión sobre el Irak de Sadam Hussein $^{52}$ dejando a Irán cierta capacidad para actuar.

En cambio, a su vuelta a Beit Aghion ${ }^{53}$, Netanyahu se encontró con un Irán en pleno desarrollo nuclear. Por ello tal y como anunció en el discurso de la Universidad de Bar-Ilán (2009), Netanyahu consideró a Irán el principal problema de seguridad de Israel, incluso por delante de Hamás o Siria. Por ello, en los 18 discursos analizados el término Iran fue utilizado un total de 538 veces, solo superado por Israel (918 veces) y por delante incluso de conceptos tan importantes como Jewish People (405) o Palestinian State (318 veces).

\footnotetext{
${ }^{52}$ Parsi, Trita (2007): Treacherous Alliance: The Secret Dealing of Israel, Iran and the United State, New Haven, Yale University Press, pp. 195-197.

${ }^{53}$ Residencia del primer ministro de Israel.
} 
Con un contexto nacional ${ }^{54}$ e internacional ${ }^{55}$ nada favorable, Netanyahu tuvo que adoptar una estrategia completamente diferente a la de 1996. Si bien es cierto que en el plano operativo (sabotajes, asesinato de científicos, virus informáticos) las acciones contra Irán se concentraron en el periodo $2010-2014^{56}$, en el plano diplomático la presión se concentró en el binomio 20142016. Por ello, las alusiones a Irán en los discursos públicos (gráfico 6) se concentraron en el periodo 2014-2016.

Gráfico 6: Alusiones a términos relacionados Irán y su Programa Nuclear.

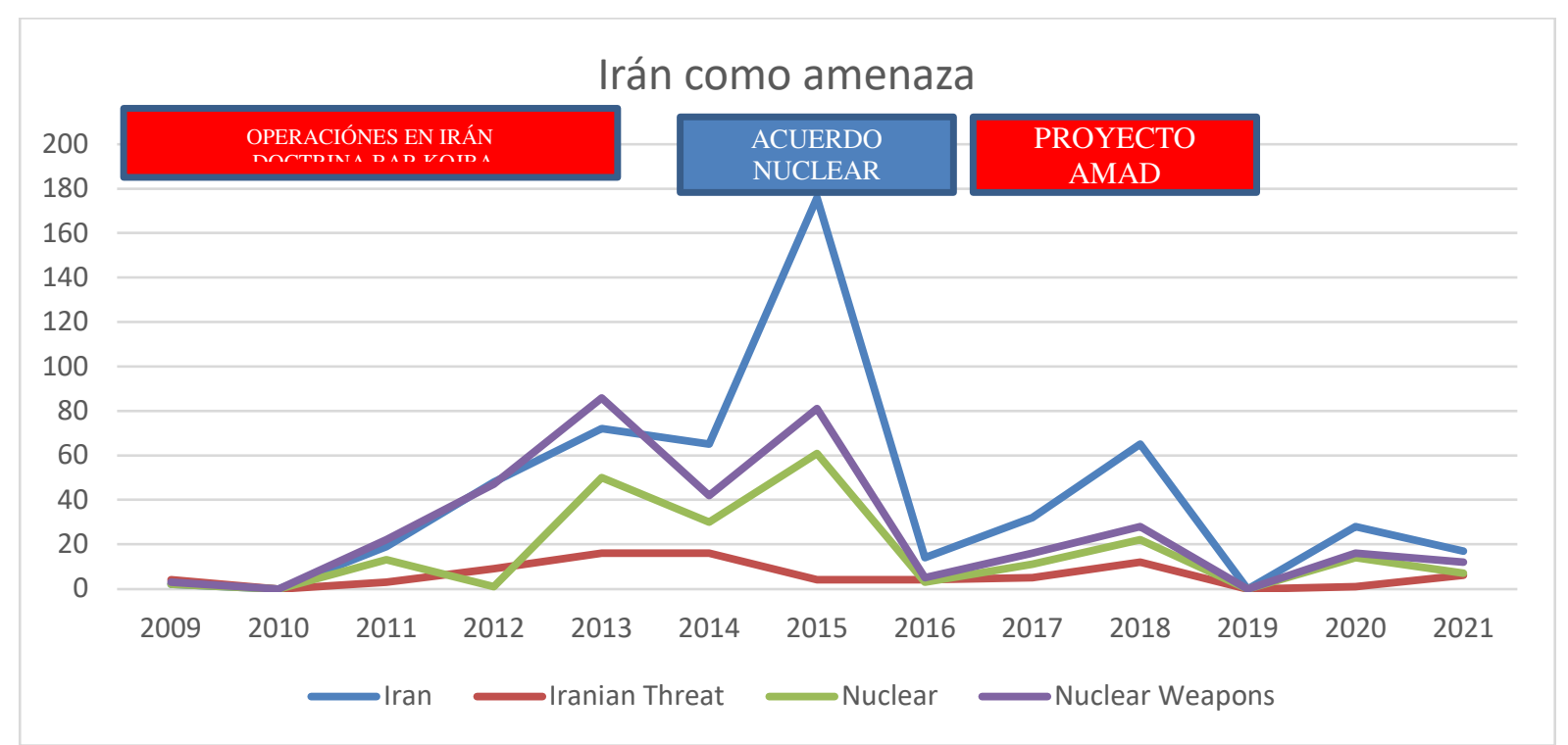

Fuente: Elaboración propia

La explicación a este fenómeno la tenemos que buscar en los esfuerzos diplomáticos llevados a cabo por Netanyahu para ralentizar el programa nuclear. El primer ministro fue muy cuidadoso con la elección de los foros a los que dirigía sus alocuciones, destacando sobre todo el Congreso de los Estados Unidos y la Asamblea General de las Naciones Unidas. En este último foro, el primer ministro llegó a sacar un dibujo de una bomba con un porcentaje del 90\% que indicaba el supuesto progreso que había alcanzado Irán en su desarrollo nuclear. Esta práctica, más propia de debates electorales que de alocuciones diplomáticas debe ser explicada por la necesidad de Netanyahu de llamar la atención internacional sobre el desarrollo nuclear de Irán. Si bien es cierto que la idea de un Irán nuclear puede resultar amenazante, algunos responsables de la seguridad de Israel como el ex director del Mossad Ephrain Halevy o el ex jefe del Estado Mayor Dan Haluz consideraron que la preocupación y sobre todo, la escenificación de Netanyahu fue una sobreactuación ${ }^{57}$.

\footnotetext{
${ }^{54}$ En el año 2011 el jefe del Mossad (Meir Dagon), el director del Shin Bet (Yuval Diskin) y el Jefe el Estado Mayor (Gabi Ashkenazi) desaconsejaron un acción directa contra Irán. Pfeffer, Anshel (2017): Bibi: The Turbulent Life and Times of Benjamin Netanyahu. London, Basic Civitas Books, p. 330 y 333.

${ }^{55}$ La llegada a la Casa Blanca de Barack Obama supuso una reconfiguración de la estrategia de Netanyahu sobre Irán.

${ }^{56}$ Durante el periodo 2010-2014 Irán sufrió diferentes agresiones (informáticas, sabotajes, asesinatos) que tenían por objetivo frenar el desarrollo de su programa nuclear. Esta estrategia ha sido denominada como Doctrina Bar Kojba. Ver Priego, Alberto (2021): "La inaplicabilidad de la Doctrina Begin en Irán: la Doctrina Bar Kojba" Documentos de Opinión IEEE, 70/2021, en

http://www.ieee.es/Galerias/fichero/docs_opinion/2021/DIEEEO70_2021_ALBPRI_Iran.pdf

${ }^{57}$ David, Steven R “Coping with an existential Threat” en Freedman, Robert O. (2019): Israel Under Netanyahu: Domestic Politics and Foreign Policy. Abingdon, Routledge.
} 
Imagen 1: Imagen del discurso de Netanyahu ante las Asamblea General de las Naciones Unidas 2012.

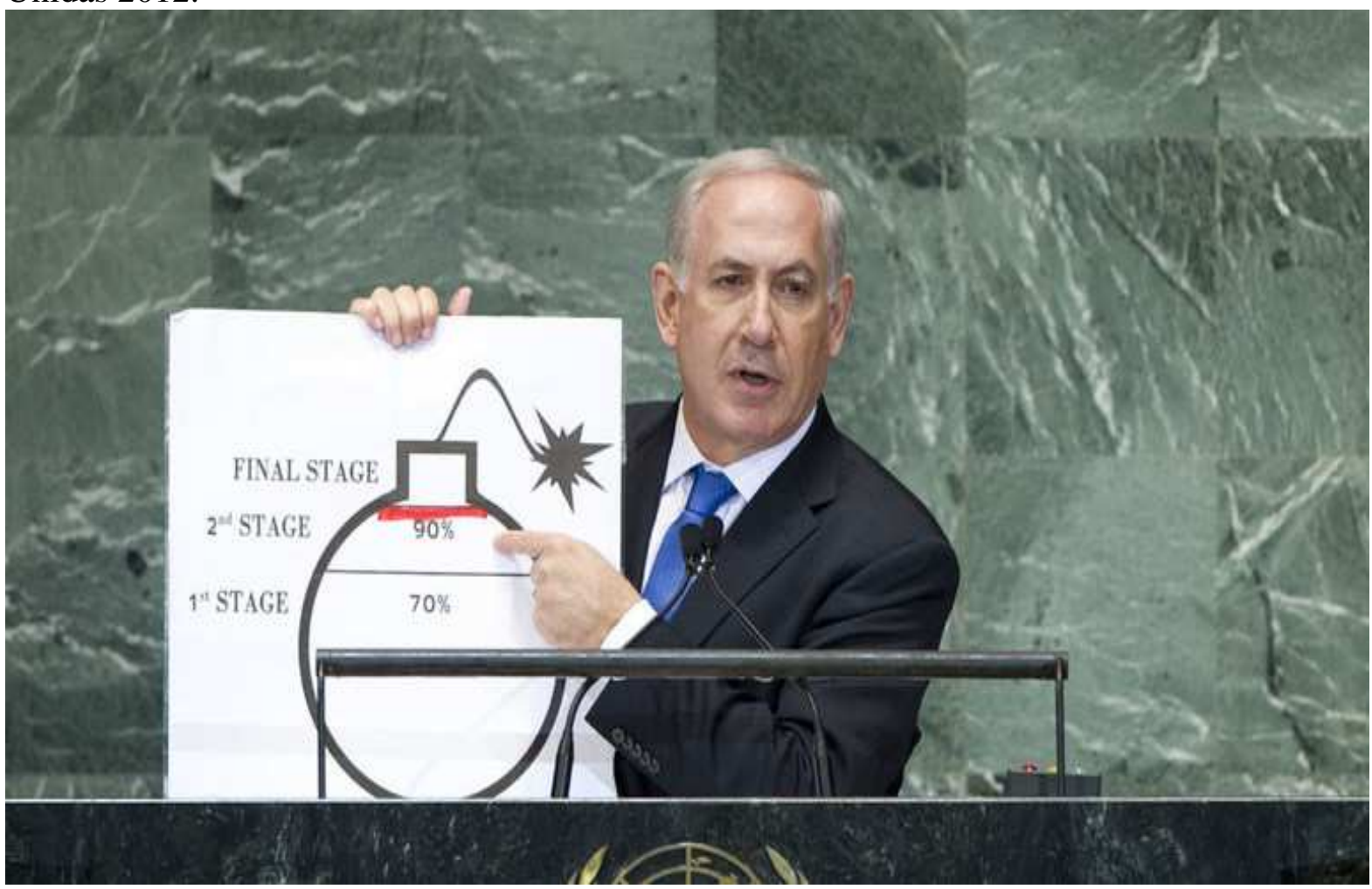

Fuente: News of the UN.

En todo caso, hechos como las negociaciones con Irán en Bagdad (2012), el acuerdo de congelación del programa de enriquecimiento (2013) y la inacción del presidente Obama en Siria (2013), provocaron que Netanyahu incrementara sus esfuerzos diplomáticos para advertir sobre la amenaza iraní. Ante la inminente firma del acuerdo nuclear (2 de abril de 2015) Netanyahu propició que el Speaker de la Cámara John Boehner le invitara para dirigirse a los representantes. Ante los representantes de Estados Unidos, Netanyahu pronunció un discurso en el que Irán fue el tema principal. En este discurso la palabra Iran aparece mencionada un total de 107 ocasiones $(0,93 \%$ del total de palabras), la palabra Nuclear $45(0,68 \%)$ y el concepto Nuclear Weapons 56 (0,85\% del total de palabras). Estos números nos muestran cual era el objetivo de Netanyahu: advertir a los representantes de Estados Unidos del peligro de un Irán nuclear.

Una vez analizada la amenaza, vamos a ver como Netanyahu ha buscado afrontarla. En este punto tenemos que hacer una distinción entre:

a) Acciones directas de carácter militar contra objetivos iraníes (científicos, equipos informáticos e instalaciones nucleares) vinculados al programa nuclear que forman parte de una doctrina (Bar Kojba) de carácter militar que complementa las acciones diplomáticas que estamos analizando.

b) Acciones diplomáticas encaminadas a la creación de alianzas con monarquías árabes sunitas para aislar regionalmente a Irán e impedir la proliferación nuclear. Como se muestra en el gráfico 7, esta estrategia que comenzó en 2011 no se hizo evidente hasta 2014. En todo caso hubo que esperar hasta 2020 para que diera sus frutos: los Acuerdos de Abraham. El estado clave en esta estrategia ha sido Emiratos Árabes Unidos, algo que se aprecia claramente al ver las 184 ocasiones que aparece mencionado en los discursos analizados. Solo Irán aparece mencionado más veces que Emiratos. 
Gráfico 7: Alusiones a las Monarquías Árabes.



Fuente: Elaboración propia

La búsqueda de nuevos aliados en el Mundo Árabe no solo tiene un efecto directo sobre Irán ya que además supone la superación del denominado "Complejo del Gueto 58 " o "Complejo de Masada 59 ". Este síndrome o complejo, se basa en el mantenimiento de un aislamiento internacional -sobre todo con los vecinos árabes- como forma de garantizar la supervivencia de Israel. Si bien es cierto que este aislamiento ha permitido que Israel sea más seguro, no es menos cierto que ha perjudicado enormemente a la reputación de Israel. Los Acuerdos de Abraham y su posterior extensión a países como Sudán o Marruecos, no solo son importantes por el acercamiento de posturas respecto de Irán, sino también porque rompen el cinturón de seguridad, protección y aislamiento establecido por el propio Israel con los países árabes.

Viendo los mapas 1 y 2, podemos afirmar que Netanyahu ha hecho de la necesidad virtud y usando la amenaza iraní como "escusa", ha revertido la relación con estados como Emiratos, Bahréin, Sudán, Marruecos o incluso Arabia Saudí convirtiendo a sus enemigos en potenciales aliados. Si bien es cierto que el origen de esta cooperación no es otra que el temor compartido hacia Irán, la normalización de las relaciones con buena parte del Mundo Árabe supone un cambio estructural en la política exterior de Israe ${ }^{60}$. Este cambio de dinámica es uno de los puntos fundamentales de la Doctrina Netanyahu, y probablemente no solo se mantendrá muchos años, sino que tendrá un efecto imitación en otros estados como Omán, Kuwait o la mencionada Arabia Saudí. Con esta nueva situación Israel podrá transformar su política exterior no solo hacia Oriente Medio sino también hace el resto del mundo ya que los Acuerdos de Abraham reducen sus amenazas en Oriente Medio permitiéndole concentrarse en otras áreas como África o Asia.

\footnotetext{
${ }^{58}$ Schilling, Christopher L. "The Ghetto Complex: Rethinking Israel's foreign policy" The International Journal of Interdisciplinary Social Science, vol. 5, nº 4 (2010).

${ }^{59}$ Masada es un símbolo del nacionalismo judío. Fue el lugar donde resistieron los últimos judíos en la Gran Revuelta Judía ante de cometer un suicidio colectivo. Muchos oficiales del Tzahal realizan su juramento en las ruinas de Masada.

${ }^{60}$ Priego, Alberto "La nueva política de Israel hacia el Golfo Pérsico” Documentos Opinión Instituto Español de Estudios Estratégicos, n’. 147 (2020). en

http://www.ieee.es/Galerias/fichero/docs_opinion/2020/DIEEEO147_2020ALBPRI_Abraham.pdf
} 
Mapa 1: Relaciones de Israel en Oriente Medio.

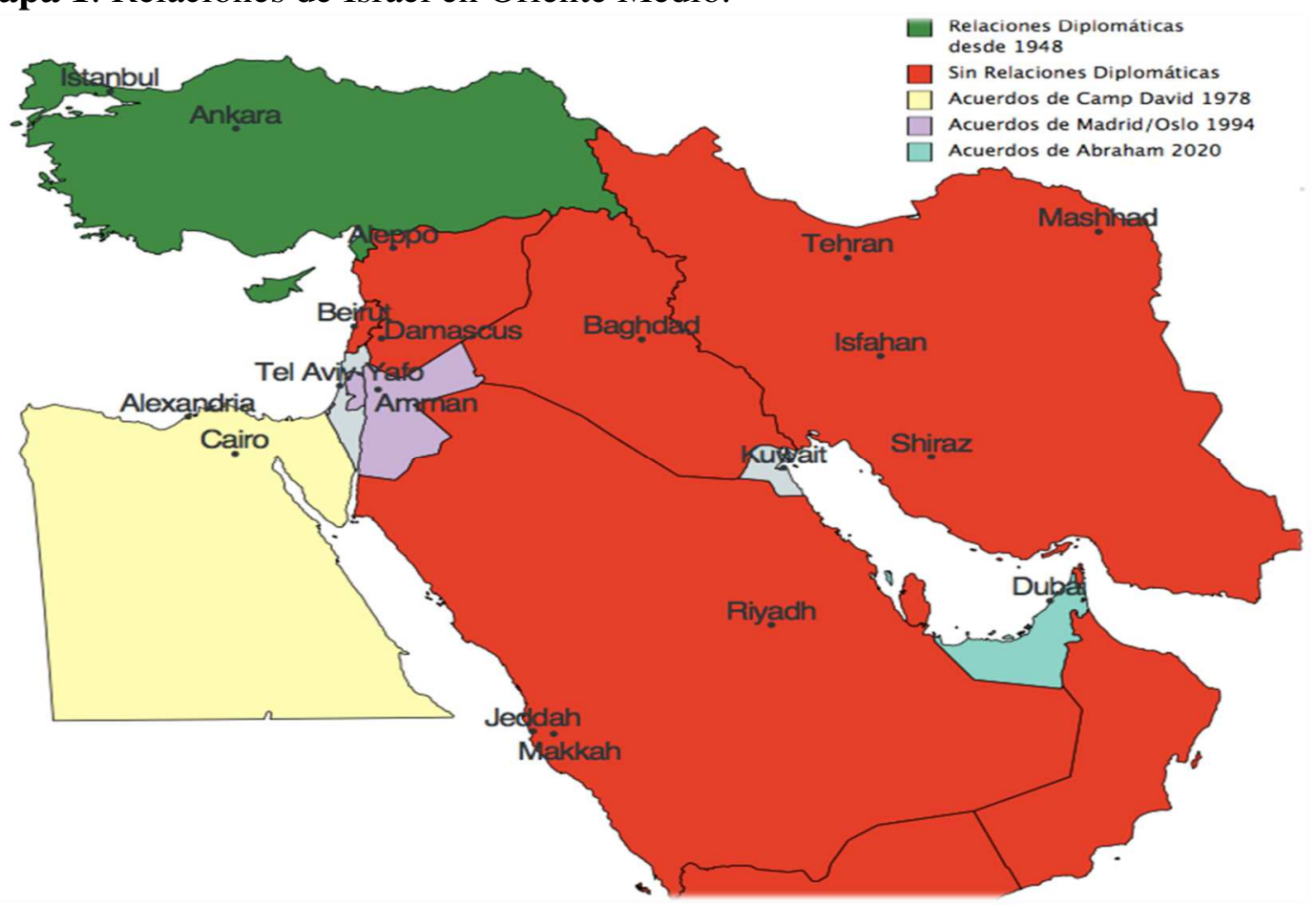

Fuente: Elaboración propia

Mapa 2: Relaciones de Israel en el Norte de África.

Sin Relaciones Diplomáticas

Efecto Acuerdos de Abraham

Acuerdos de Camp David 1978

Acuerdos Mauritania-Israel 1999

Fuente: Elaboración propia

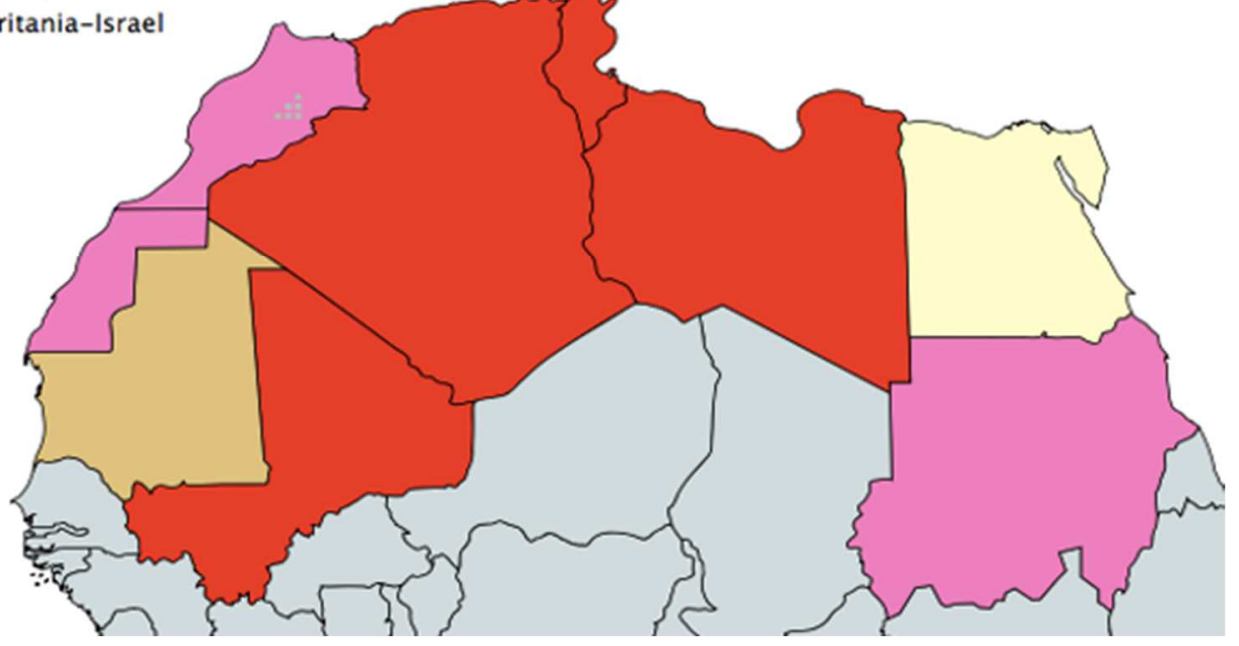

\subsection{Pilar Económico (Crisis Financiera)}

Netanyahu se define a sí mismo como un convencido de la economía de mercado y como un ferviente seguidor del pensamiento económico neoclásico61. Esta afirmación no es una mera

${ }^{61}$ Nathason, Roby and Weiss, Yanai "The Israeli economy under the leadership of Netanyahu. Growth and stability at the cosrt of weakening the welfare state" en Freedman, Robert O. (2019): Israel Under Netanyahu: Domestic 
declaración ya que tanto en los años en los que Netanyahu fue ministro de finanzas (2003-2005) como posteriormente en los años que a ejerció como primer ministro (2009-2021), Netanyahu apostó por medidas económicas muy liberales. Sin embargo, la economía no solo no ha sido el principal campo de desarrollo profesional de Netanyahu, sino que en su estrategia de gobierno la economía siempre ha tenido un componente adjetivo y no sustantivo. En otras palabras, a pesar de su formación y de haber sido ministro de finanzas, para Netanyahu la economía ocupa un papel subordinado tanto a la seguridad como a la diplomacia y, por tanto, la economía es un mero instrumento al servicio de las otras dos prioridades.

En todo caso, debemos destacar el interés de Netanyahu por la economía en general y por la liberal en particular, algo que se puso de manifiesto en el discurso de Bar Ilán donde Netanyahu, identificó a la crisis económica como una de las tres prioridades que en aquel momento tenía Israel. Para superar ese reto, el primer ministro adoptó un plan basado en tres puntos que no solo servirá para la superación de la crisis, sino también para transformar la economía de Israel:

- La liberalización económica

- La apuesta por la tecnología y la innovación

- La exportación de energía.

Liberalización económica. De forma general, podemos decir que el modelo económico de Netanyahu fue bastante exitoso ya que, durante los años que Netanyahu fue primer ministro, Israel logró un crecimiento medio del 3,7\% con picos de crecimiento que rozaron incluso el $6 \%$ del PIB. Al igual que ocurrió en otros lugares, este crecimiento solo se vio truncado por la llegada de la pandemia en 2020. Sin embargo, el modelo económico tuvo bastante contestación por disparar las desigualdades entre los israelíes.

Gráfico 8: El crecimiento económico de Israel entre 2009 y 2021.

\section{Crecimiento Económico de Israel}

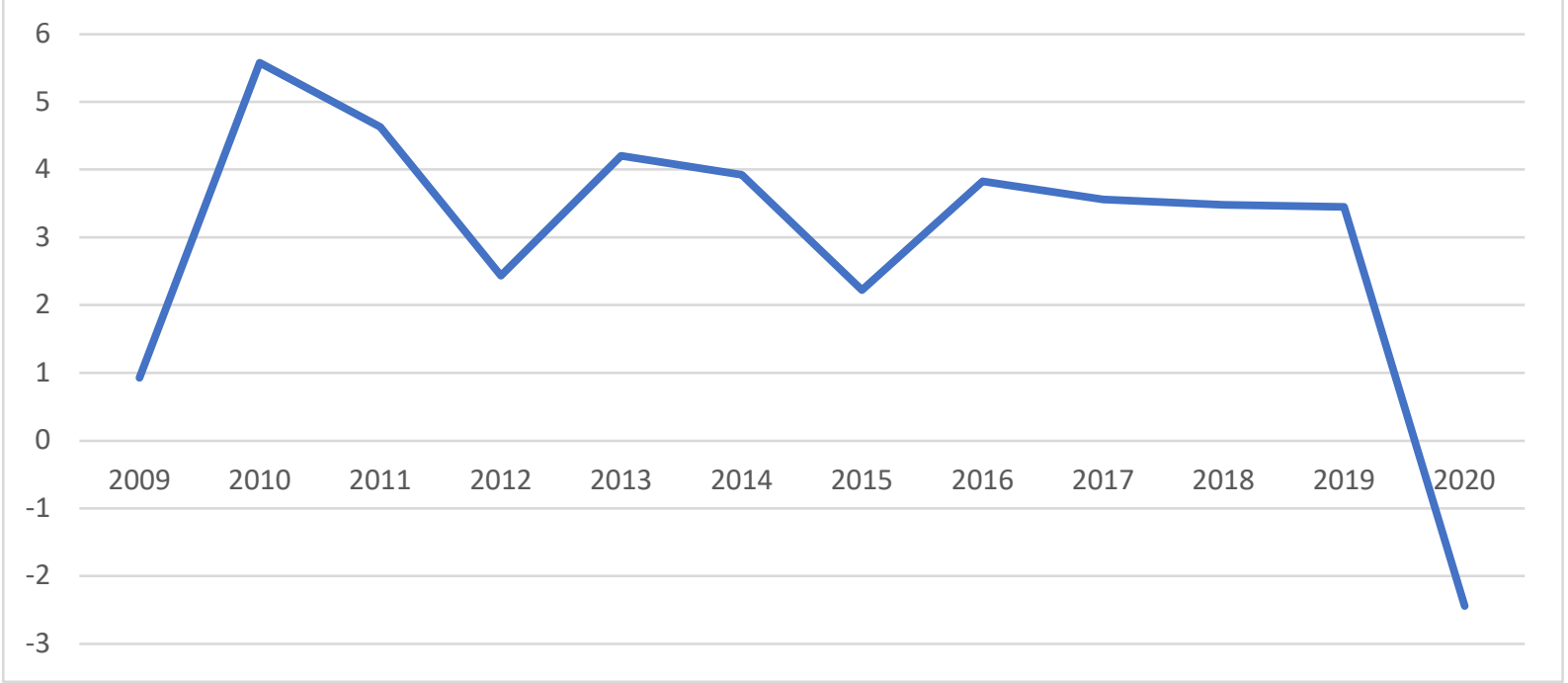

Fuente: Banco Mundial

Además, Netanyahu logró superar uno de los principales problemas de la sociedad israelí: el desempleo. Cuando el líder del Likud llegó al poder la tasa de paro rozaba el 10\% (Ver Gráfico 9) y antes de la pandemia rondaba pleno empleo (3,8\% en 2019). Sin embargo, hay que decir

Politics and Foreign Policy. Abingdon, Routledge. 
que Israel ha sido una de las economías que más han sufrido con la COVID-19 aunque este dato no debería empañar el esfuerzo hecho en los años anteriores.

Gráfico 9: Tasa de desempleo en Israel 2009-2021

\section{Desempleo}

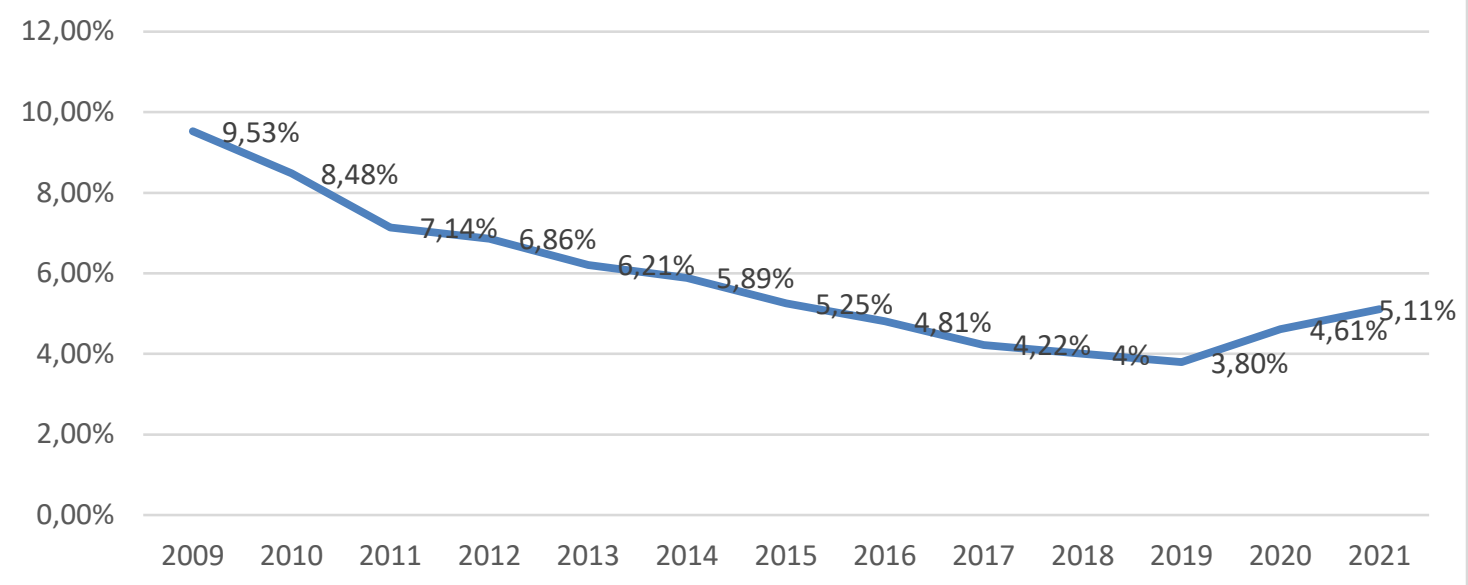

Fuente: Ministerio de Trabajo de Israel.

Si analizamos el primero de los puntos de su proyecto económico -la liberalización- podemos afirmar sin miedo a equivocarnos que la economía israelí hoy es más libre que cuando Netanyahu llegó al poder. De hecho, no solo es más libre, sino que la economía israelí está entre las más libres del mundo, ocupando el puesto 26 (Economic Freedom index Heritage Foundation) lo que implica que durante el mandato Netanyahu Israel recuperó 16 puestos (gráfico 10).

Gráfico 10: Liberalización de la Economía Israelí.

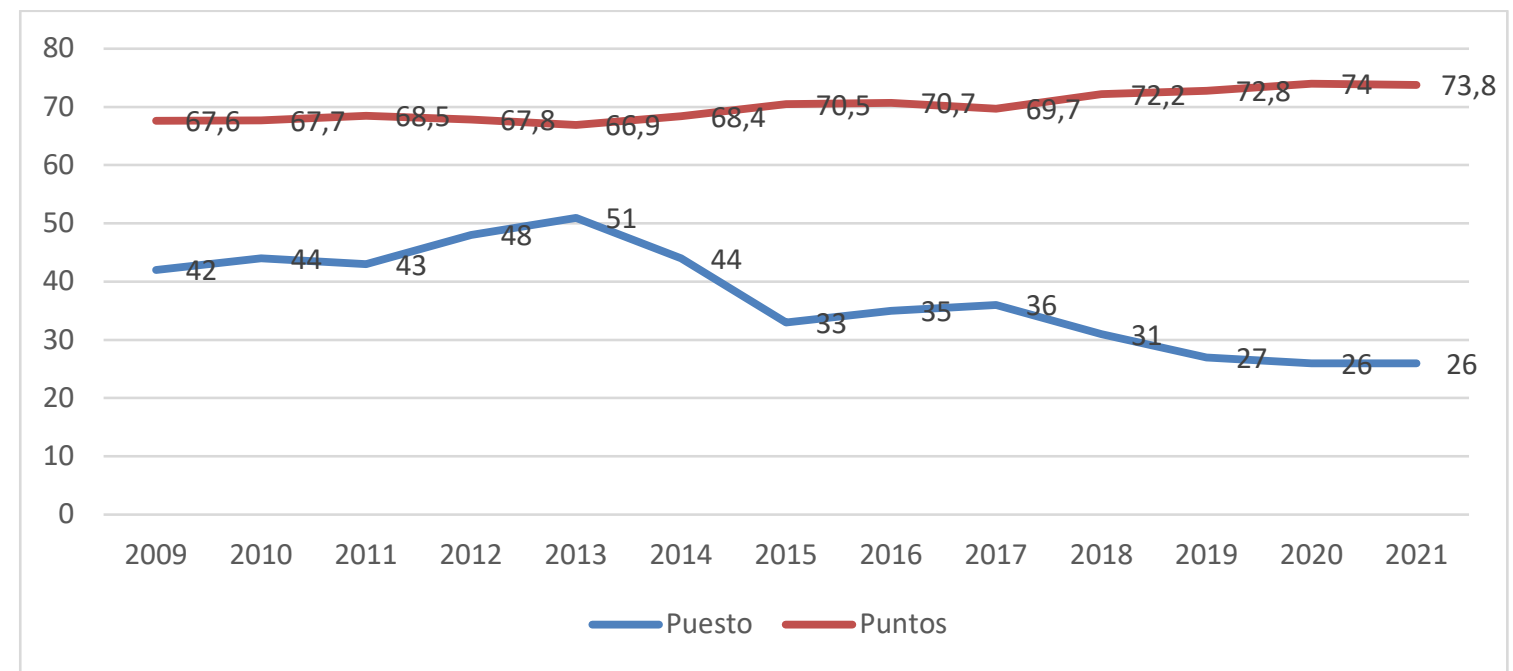

Fuente: Economic Freedom Index - Heritage Foundation

Uno de los aspectos en los que se aprecia esta liberalización, es en la presión impositiva. Cuando Netanyahu fue nombrado ministro de finanzas (2003), el impuesto de sociedades estaba en un $36 \%$, y tan solo dos años más tarde, descendió al 23\%. En los años que estuvo en la oposición, la tasa impositiva volvió a subir hasta el 34\%. Sin embargo, en 2018 Netanyahu lo volvió a rebajar hasta el $23 \%$. Este descenso en la presión impositiva tiene como principal objetivo la atracción de inversión extranjera, sobre todo para las empresas de alta tecnología que son para Netanyahu una prioridad. 
Gráfico 11: Alusiones económicas en los discursos de Netanyahu

\section{conceptos económicos}

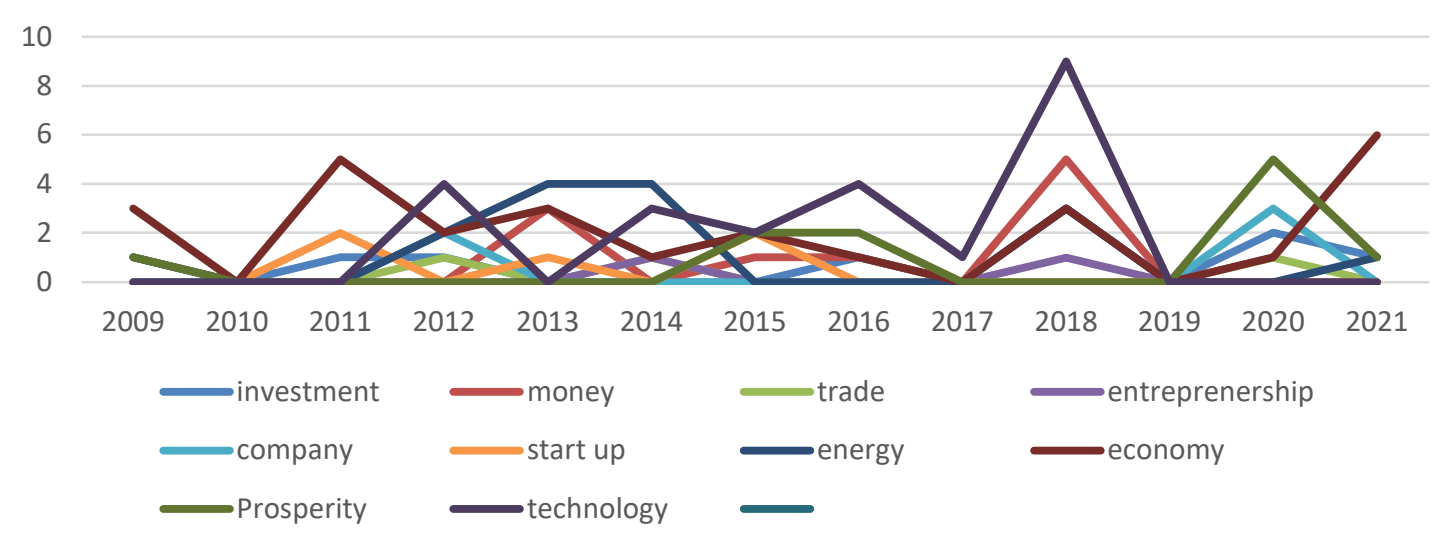

Fuente: Elaboración propia

Tal y como se aprecia en el gráfico 11, los conceptos económicos más usados por el primer ministro, además de la mencionada tecnología, son los relacionados con la energía. Dejando a un lado que resulta evidente que estos dos sectores son una parte muy importante de la transformación económica emprendida por Netanyahu ${ }^{62}$, tanto la tecnología como la energía poseen para Israel otros beneficios que no son puramente económicos.

Tecnología: La ciencia ${ }^{63}$ y la tecnología se han convertido en uno de los principales activos de la diplomacia pública israelí. Desde el gobierno de Netanyahu se han realizado verdaderos esfuerzos para vincular el nombre de Israel con el de las start-up. Incluso existe un equipo profesional ciclista apoyado por el estado de Israel, que lleva por nombre Israel Start-Up Nation $^{64}$. Este aspecto ha sido uno de los principales activos en la gran mejoría que ha experimentado la reputación internacional de Israel, algo que vemos en el gráfico 12.

Gráfico 12: Reputación Internacional de Israel (2010-2018).

\section{Reputación Internacional}

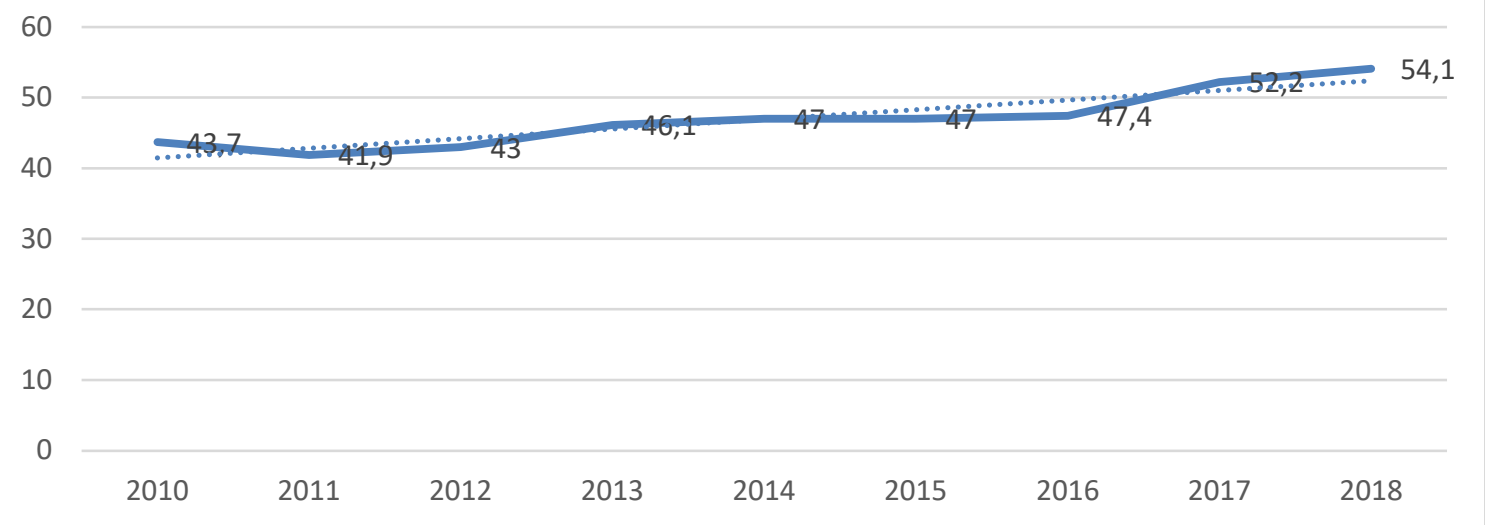

Fuente: National Reputation Ratings

\footnotetext{
${ }^{62}$ Israel posee 63 empresas en el Nasdaq, siendo el primer país del mundo en número.

${ }^{63}$ Israel es el cuarto país del mundo en producción científica. Ilani, Ofri (2009): "Israel ranks fourth in the world in scientific activity, study finds". Haaretz, 17 November 2009.

${ }^{64}$ Sobre esta relación ver Senor, Dan and Singer, Saul (2009): Start-up Nation: The Story of Israel's Economic Miracle, Twelve, New York.
} 
La mejora de la reputación de Israel ha resultado clave para la normalización de sus relaciones con las monarquías sunitas ${ }^{65}$, un proceso que no solo tiene un beneficio económico, sino que tiene por principal objetivo frenar las aspiraciones nucleares de Irán.

Energía: El tercer pilar de la estrategia económica de Netanyahu es la energía. Entre los años 2009 y 2012 Israel descubrió importantes yacimientos de gas natural (Tamar y Leviathan) que le permitieron pasar de ser importador a ser exportador de energía. Este hecho tuvo dos importantes consecuencias:

- No dependía de nadie para la producción de electricidad, lo que le permitía tener independencia en su política exterior

- Sus vecinos, Egipto y Jordania, se convirtieron en receptores netos de energía israelí, lo que ha reforzado la vigencia de los acuerdos de Camp David y Oslo respectivamente. En el caso de Egipto, este cambio fue especialmente significativo ya que hasta $2010 \mathrm{el}$ país del Nilo era un importante suministrador de gas natural a Israel.

Para concluir este apartado podemos afirmar que la transformación económica emprendida por Netanyahu fue más compleja de lo que parecía. Si bien es cierto que tuvo la función manifiesta de superar la crisis económica, no es menos cierto que oculta una función latente que consistía en mejorar la maltrecha imagen de Israel, un aspecto imprescindible para que los Acuerdos de Abraham vieran la luz. Además, Netanyahu ha apostado por el desarrollo del sector energético convirtiendo a Israel en un importante exportador de energía, sobre todo en lo que se refiere a sus dos vecinos Jordania y Egipto. La relación económica intensa evitará los roces de Jerusalén con Ammán y el Cairo, lo que supone un seguro para los acuerdos de Camp David y Oslo.

\subsection{Pilar Diplomático (Proceso de Paz)}

Desde un punto de vista personal, Benjamín Netanyahu está lejos de ser un político favorable a cualquier proceso de paz con los palestinos. De hecho, la primera vez que se convirtió en primer ministro, lo hizo gracias a las duras críticas vertidas contra los Acuerdos de Oslo. Así, en la campaña electoral del 1996, Netanyahu se presentó como "Mr. Security" y cuando alcanzó el poder, con los acuerdos ya firmados, hizo todo lo posible para evitar su puesta en práctica. Por ello, a nadie extrañó que el Enviado Especial para Oriente Medio de Estados Unidos calificara a Netanyahu como alguien que "no tenía ningún interés en la búsqueda de la paz"

Además de su oposición a los Acuerdos de Oslo, Netanyahu nunca ocultó su discrepancia con los planes de desconexión de la Franja de Gaza llevados a cabo por el primer ministro Ariel Sharon. Esta discrepancia le llevó incluso a abandonar el ejecutivo y a convirtirse en 2005 en el líder de la oposición. Durante este periodo de cuatro años en los que estuvo fuera del gobierno, Netanyahu criticó activamente el rol de mediador de Washington llegando a afirmar que sus esfuerzos por acercar posturas eran una "verdadera pérdida de tiempo ${ }^{67 "}$. Sus discrepancias no solo eran sobre la opción de comprometerse a crear dos estados, sino que alcanzaban la propia metodología del proceso de paz. Netanyahu, apostaba por cambiar la

\footnotetext{
65 Suleiman, Mohammed (2021): "How tech is cementing the UAE-Israel alliance" MEIA75, en https://www.mei.edu/publications/how-tech-cementing-uae-israel-alliance.

66 "Neither President Clinton nor Secretary Albright believed that Bibi had any real interest in pursuing peace." Beinart, Peter: "How U.S. Jews Stymie Peace Talks", The Daily Beast, 27 September 2010, en http://www.thedailybeast.com/articles/2010/09/27/us-jewish-groups-help-make-palestinian-stateimpossible.html.

${ }^{67}$ Schneider, Howard: "Poll Gives Netanyahu Positive Marks Despite Rift with US". The Washington Post, 20 March 2009
} 
lógica de "Paz Por Territorios" que había inspirado Oslo, por lo que el denominó la "Paz Económica" $"$.

La propuesta de la "Paz Económica" fue presentada en sociedad en el discurso de Bar Ilán, está basada en una aproximación funcionalista que busca la promoción económica y el bienestar en determinados lugares de Cisjordania, para extender posteriormente el modelo al resto del territorio palestino. En otras palabras, lo que Netanyahu buscaba, era convencer ${ }^{69}$ a los palestinos de que les compensaba aceptar cesiones aparentemente inasumibles desmilitarización de Palestina, Jerusalén como la capital indivisible de Israel o el no retorno de "refugiados palestinos" - a cambio de una política económica muy generosa con su población.

Si bien muchos pensaron que esta "Paz Económica" había muerto en 2011, la estrategia de Netanyahu era más compleja de lo que parecía ya que, se extendió hasta 2020. Para entenderla mejor la hemos dividido en tres fases:

a) Fase Uno (2009-2011). Presentación de su modelo funcionalista de paz que hemos denominado "Paz Económica".

b) Fase Dos (2012-2019) Adormecimiento tanto de su propuesta de "Paz Económica" como de cualquier negociación con los palestinos hasta que se lograran las condiciones económicas y diplomáticas adecuadas.

c) Fase Tres (2020-2021). Recuperación y relanzamiento de su propuesta de "Paz Económica" transformada en el denominado Plan Trump.

Gráfico 13: Alusiones a conceptos relacionados con Proceso de Paz y con los palestinos.



Fuente: Elaboración propia

\footnotetext{
68 Ahren, Raphael "Netanyahu: Economics, Not Politics, Is the Key to Peace" 20 November 2008,.en https://www.haaretz.com/1.5061173.

69 "Economic peace is not a substitute for peace, but it is a very important component in achieving it" Netanyahu, Benjamin (2009): "Israeli PM Netanyahu's Bar-Ilan Speech" p. 2., en https://ecf.org.il/media_items/1141
} 
Gráfico 14: Alusiones a conceptos relacionadas con Palestina

\section{Palestina}

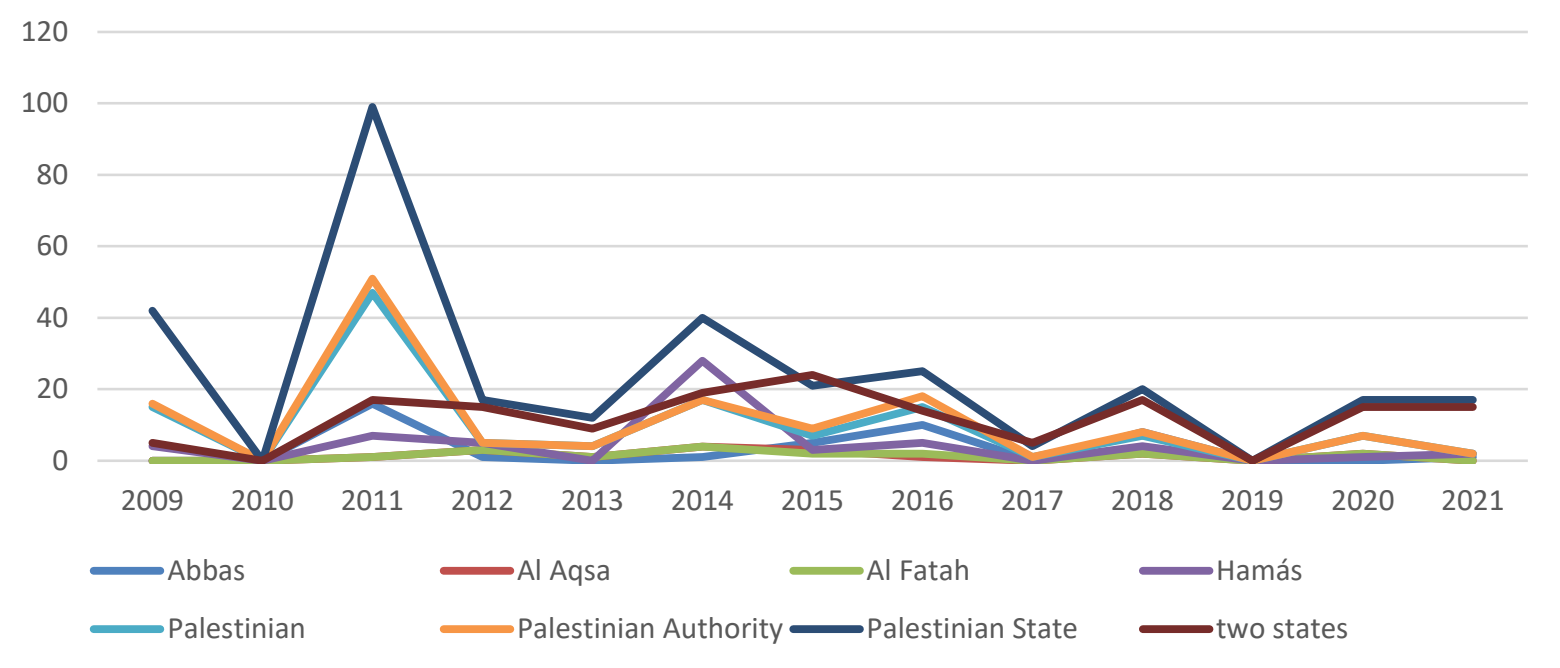

Fuente: Elaboración Propia.

Tal y como vemos en los gráficos 13 y 14, las alusiones tanto a conceptos relacionados con procesos de paz y como al propio problema palestino, han seguido la misma lógica temporal. En los dos primeros años de gobierno de Netanyahu, hay una narrativa centrada en estas dos cuestiones (Fase 1), para desaparecer después durante unos 7 años (Fase 2). Finalmente, en el año 2020 (Fase 3) vuelve a aparecer en la agenda del primer ministro para desaparecer definitivamente en 2021.

La pregunta que cabe hacerse es por qué Netanyahu adormeció este tema durante estos siete años para recuperarlo después. La respuesta la debemos buscar en dos puntos que ya hemos tratado anteriormente.

a) La necesidad de lograr una recuperación económica que le permitiera poder sustanciar económicamente su propuesta de paz con los palestinos.

b) La necesidad de crear las condiciones necesarias para propiciar el acercamiento a las monarquías sunitas que le permitiría por un lado frenar regionalmente a Irán y por el otro aislar a los palestinos ${ }^{70}$.

Durante esta segunda fase que hemos denominado "años de adormecimiento", ocurrieron algunos hechos que complicaron la ejecución de la estrategia de Netanyahu como el cambio de estatus de la Autoridad Nacional Palestina en la Asamblea General de la ONU (2012), las operaciones en Gaza $(2012,2014)$ o las protestas palestinas en Jerusalén (2018) Sin embargo, si bien es cierto que estos hechos supusieron un percance significativo, ninguno de estos asuntos fue lo suficientemente grave como para derribar la estrategia de Netanyahu.

Finalmente, en enero 2020 - muy avanzada la negociación de los Acuerdos de Abraham - se presentó el Plan Trump ${ }^{71}$ (Peace to Prosperity: A Vision to Improve the Lives of the Palestinian and Israeli People) una iniciativa que no solo siguió la lógica de la "Paz Económica" de Netanyahu, sino que además mantuvo las condiciones planteadas a los palestinos por el primer ministro israelí en su discurso de Bar Ilán en 2009.

\footnotetext{
${ }^{70}$ Entre los años 2010 y 2021 no se produjo ningún encuentro público de alto nivel entre israelíes y palestinos.

${ }^{71}$ Sobre el Plan Trump ver Priego, Alberto (2020): "El plan Trump: seguridad a cambio de bienestar" Docuemnto de Opinión IEEE, 18/2020 (9 de marzo), en

http://www.ieee.es/Galerias/fichero/docs_opinion/2020/DIEEEO18_2020ALBPRI_planTrump.pdf
} 
Mapa 3: El Valle del Jordán en el Plan Trump.

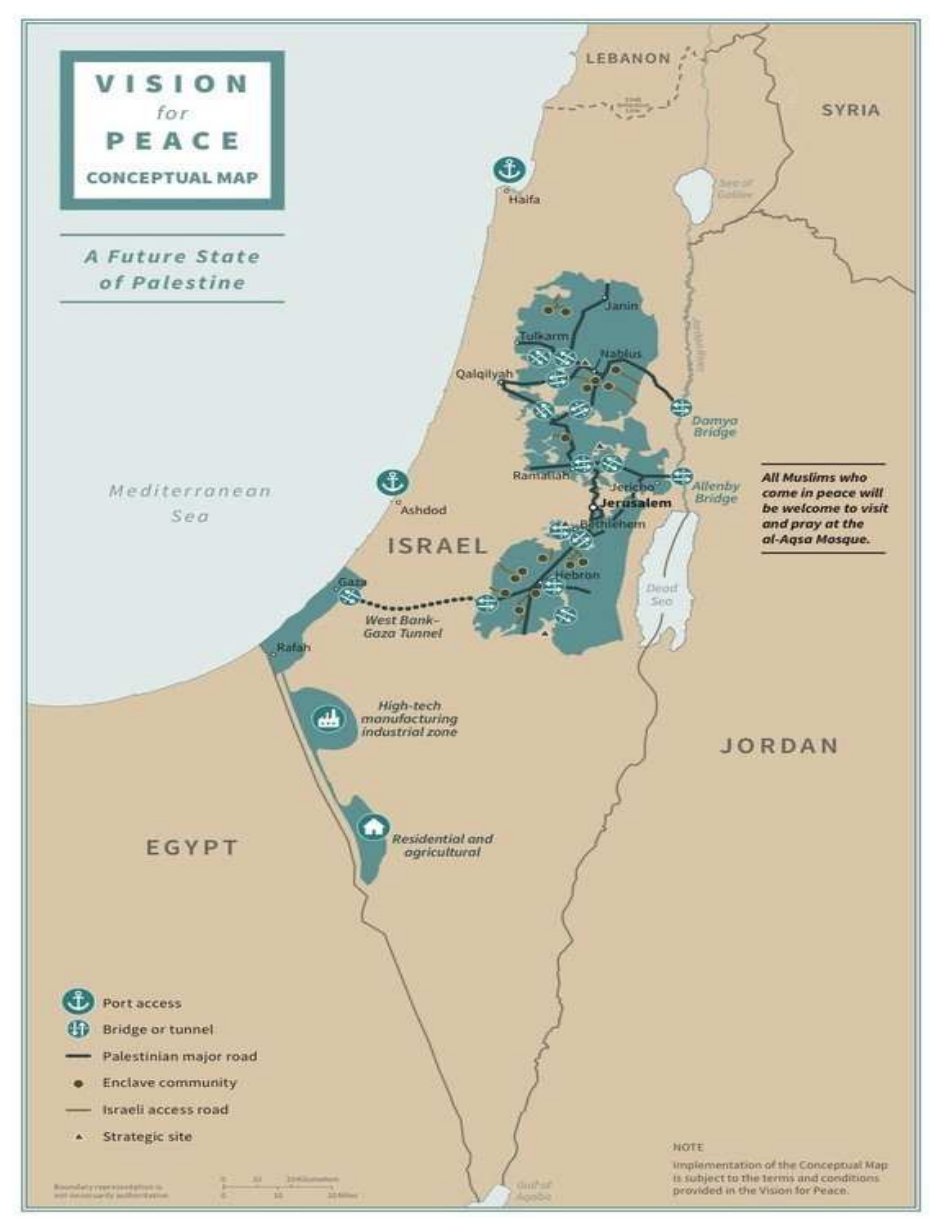

Fuente: The White House

Un punto de especial interés del Plan Trump, fue la inclusión del Valle del Jordán dentro un territorio que quedaría bajo control Israel. Esta cuestión fue ya planteada por Netanyahu en la campaña electoral de 2019 lo que muestra la confluencia entre los planteamientos del primer ministro y la propuesta del presidente de los Estados Unidos.

Si bien, las condiciones y las propuestas para los palestinos eran prácticamente las mismas $^{72}$, lo que difiere entre el Plan Trumo y los planteamientos iniciales de Netanyahu fue la situación de la Autoridad Nacional Palestina. Si bien en el año 2009 la Autoridad Nacional Palestina mantenía el apoyo del Mundo Árabe, en el año 2020 Ramala había perdido el apoyo de Arabia Saudí, de Emiratos Árabes Unidos, de Bahréin, de Sudán y de Marruecos, al salirse estos Estados de "los tres noes"73" de Jartum. Así, gracias a la Doctrina Netanyahu, no solo se cambia la lógica de paz por territorios a paz por prosperidad, sino que además reduce sustancialmente los apoyos de los palestinos en el Mundo Árabe, debilitando por tanto su posición en futuras negociaciones de paz. Este hecho supone un cambio estructural en la política exterior de Israel y parece seguro que cambiará la posición de Jerusalén en el plano regional. También cabe destacar que la coincidencia entre el Plan Trump en otros aspectos como la consideración del futuro palestino como un "Minus State" o el mantenimiento de los principales asentamientos.

\footnotetext{
${ }^{72}$ Algunos aspectos como la construcción de un corredor entre Cisjordania y Gaza no están recogidas del pensamiento de Netanyahu sino del Plan Olmert

${ }^{73}$ Los tres noes de Jartum eran: no a la paz con Israel, no al reconocimiento y no a la negociación hasta que se volviera a las fronteras de 1967.
} 


\section{Conclusiones}

El primer elemento que debemos destacar de la Doctrina Netanyahu es el componente personal. Benjamín Netanyahu es una persona con unas experiencias personales -la muerte de su hermano "Yonni" en la Operación Entebbe, la relación de su padre con Zeev Jabotinski o su especial relación con Estado Unidos- que han marcado su forma de hacer política. Estas experiencias han provocado el desarrollo de una fuerte personalidad que tiene un reflejo claro en lo que hemos denominado Doctrina Netanyahu.

Dentro de estas experiencias tenemos que destacar su formación en los Estados Unidos, especialmente en la escuela de negocios Sloan de MIT. Debido a esta formación, Netanyahu ha desarrollado una forma neo-liberal de entender la economía, forma que a la postre, ha marcado su carrera política. Sin embargo, si bien es cierto que su formación académica es económica y que ha desarrollado puestos de responsabilidad financiera, para Netanyahu la economía no es más que un instrumento necesario para alcanzar otras prioridades. Por ejemplo, me estoy refiriendo a la paz con los palestinos o a la normalización de las relaciones con los árabes. En ambos casos, la economía se ha convertido en un instrumento de gran utilidad para desarrollar sus propuestas, lo que ha venido de la mano de una cierta de-securitización del discurso. Si bien es cierto que los Acuerdos de Abraham han sido su iniciativa más exitosa, en el caso de las propuestas de paz con los palestinos habrá que esperar al futuro para ver si dan o no sus frutos. Por lo tanto, un elemento propio de la Doctrina Netanyahu es el uso de la prosperidad económica como un elemento subordinado a la política exterior y de seguridad.

En segundo lugar, debemos mencionar la apuesta por la seguridad como forma de prevenir las amenazas apocalípticas. En este punto, la estrategia de Netanyahu tenía una doble vertiente.

- $\quad$ Por un lado, Netanyahu mantiene la línea marcada por sus antecesores del sionismo revisionista (Begin y Olmert) y apuesta por los ataques preventivos para evitar el desarrollo nuclear en países vecinos. Menachem Begin marcó el camino con la Operación Ópera en Irak (1981), Olmert lo continuó con la Operación Huerto en Siria (2007) y Netanyahu, en la medida de las posibilidades, lo ha mantenido en Irán (2010-2014 y 2020) aunque ha tenido que alterar la Doctrina Begin. En otras palabras, Mientras que Begin y Olmert pudieron lanzar ataques aéreos contra las centrales nucleares de Osirak (Irak) y Al Kivar (Siria), Netanyahu ha tenido que conformarse con la eliminación de algunos de los principales científicos del programa, con la infección de virus en sus redes informáticas y con el sabotaje de las principales instalaciones nucleares iraníes. Es decir, que Netanyahu al contrario de lo que hicieron Begin y Olmert no ha podido "solucionar" el problema y tan solo ha podido retrasarlo.

- Por otro lado, y probablemente como consecuencia de lo anterior, Netanyahu tejió una tela de apoyos internacionales en el mundo suní para aislar regionalmente a Irán y reducir así sus posibilidades de éxito. Esa tela comenzó a tejerse en agosto de 2020 en Washington con los Acuerdos de Abraham, pero ha continuado con acuerdos bilaterales con Sudán y Marruecos. No obstante, el acercamiento de los últimos meses de Arabia Saudita y Emiratos Árabes Unidos a Irán y de diversos Estados de la Liga Árabe al régimen de al-Assad, unido a la progresiva retirada de Estados Unidos de Oriente Medio significan una clara reconfiguración del escenario de Oriente Medio que sólo presuponía en parte el gobierno de Netanyahu. 
Los acuerdos con las monarquías suníes no solo tendrán un efecto directo en las relaciones con Irán, sino también se notarán en las relaciones con los palestinos. La normalización de las relaciones con el mundo sunita -Egipto (1979), Jordania (1994), Mauritania (1999), EAU, Bahréin, Marruecos y Sudán (2020)- va a debilitar mucho a los palestinos al perder estos su principal apoyo económico y político. La primera muestra se produjo en la última escalada entre Israel y Hamás con declaraciones muy críticas de Emiratos Árabes Unidas con el grupo palestino. Por ello, los Acuerdos de Abraham suponen ante los ojos de los palestinos una traición a su causa, ya que los firmantes abandonan el compromiso de los tres noes de Jartum. Así, la ruptura del consenso de Jartum también puede ser considerado como uno de las consecuciones de la Doctrina Netanyahu.

En verano de 2021 se produjo la salida de Netanyahu del gobierno de Israel. Su sucesor y en otro tiempo amigo ${ }^{74}$ y colaborador, Naftalí Bennet, parece que va a mantener muchas de las aportaciones hechas por Bibi. La normalización de las relaciones con los árabes, la aplicación de la Doctrina Bar Kojba con Irán y la apuesta por la economía como principal incentivo para negociar con los palestinos, son solo algunos de los aspectos de la Doctrina Netanyahu que ya se aprecian en el gobierno de rotación Bennet-Lapid.

Otro aspecto a tener en cuenta, aparte de la consolidación de al-Assad en Siria y el cambio de política estadounidense, es la situación en la que queda Afganistán tras la salida de las tropas norteamericanas y su impacto en Oriente Medio. No solo la situación regional va a ser más inestable, sino que, además, la credibilidad de los Estados Unidos como actor regional está cuanto menos en entredicho. Esta percepción se verá reforzada en los próximos años cuando Estados Unidos complete su progresiva retirada de Irak. Con un Oriente Medio sin una presencia norteamericana sólida y la decreciente fiabilidad de Turquía dentro de la OTAN, Israel tendrá que buscar sus propias garantías de seguridad en la zona y un mayor acercamiento a Moscú. En esta línea también incide la progresiva mejoría de las relaciones entre Siria y Jordania y la situación en el Líbano, con una decreciente influencia de Arabia Saudita.

Para concluir, podemos afirmar que (ANEXO 1), la Doctrina Netanyahu poseía tres pilares: seguridad, economía y diplomacia. Estos pilares son completivos y asimétricos ya que la economía ocupa un lugar subordinado a los otros dos y la seguridad destaca por encima del resto. Dentro de algunos años estaremos en mejor disposición de comprobar si sus postulados, realizados en un contexto internacional claramente diferente del de finales de 2021, han sobrevivido en el tiempo y que efecto han tenido para la supervivencia y la prosperidad de Israel.

\footnotetext{
${ }^{74}$ De hecho, un hijo de Bennet lleva el nombre del hermano de Netanyahu -Yonni- muerto en la Operación Entebbe
} 


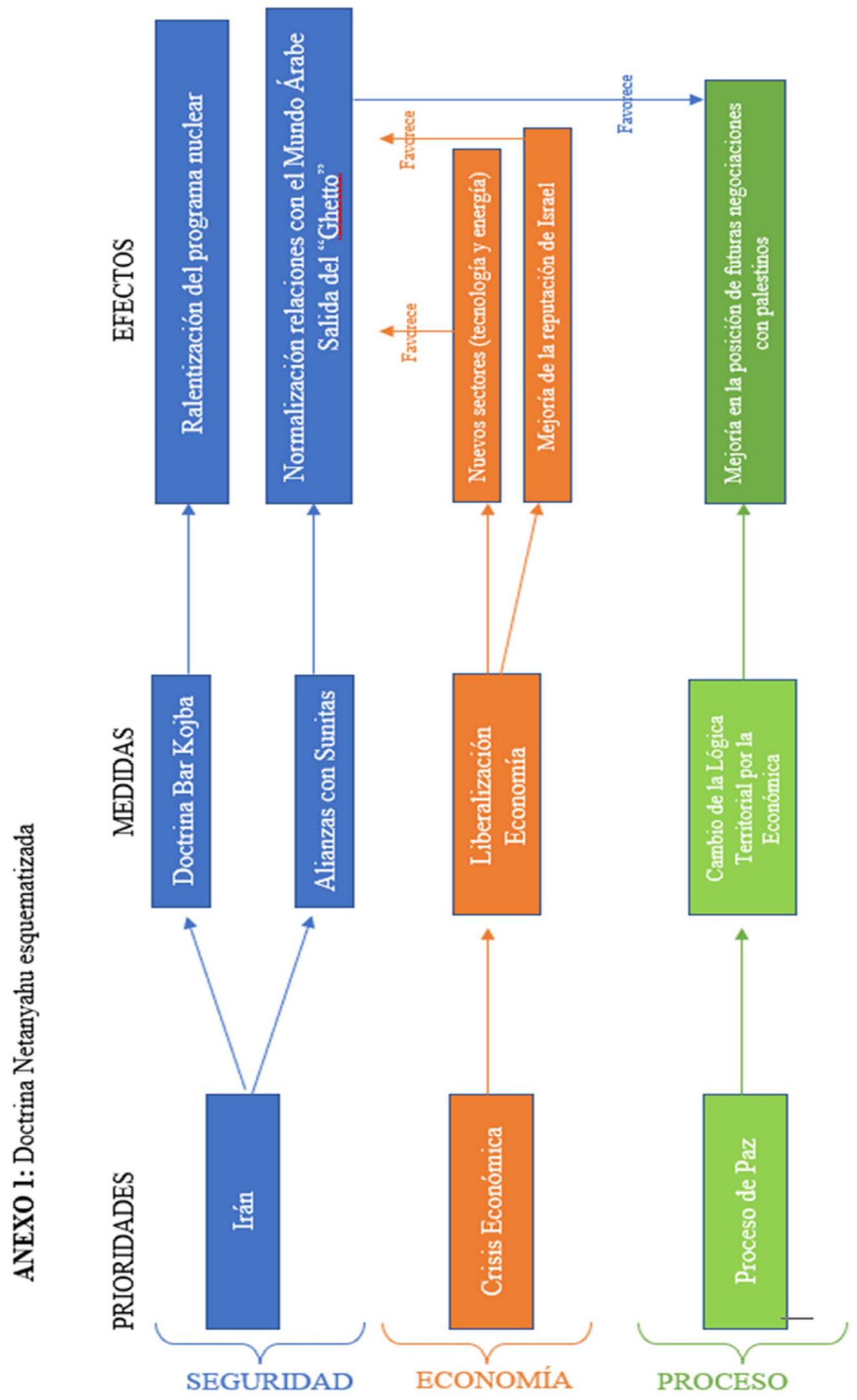


产 కై कू nबे 000000000000000000000

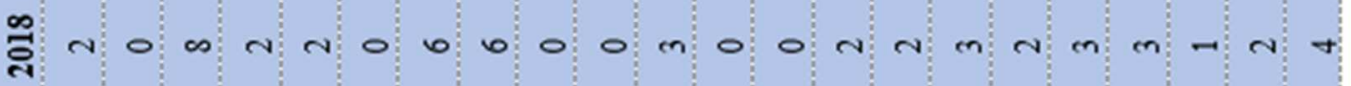

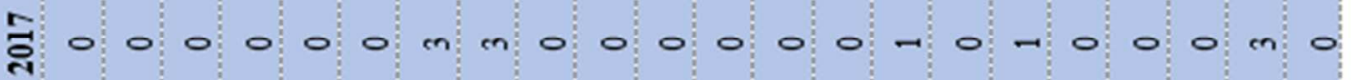

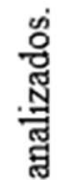
สี ติ בై ले ฮี้

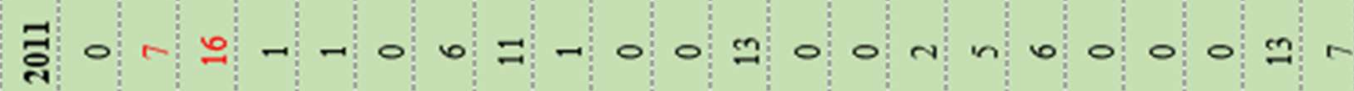

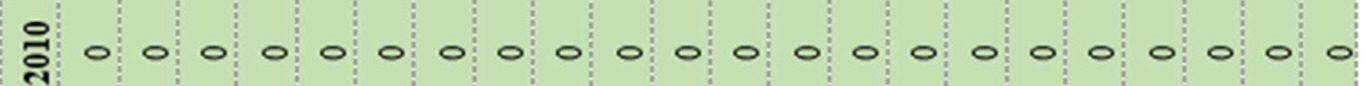

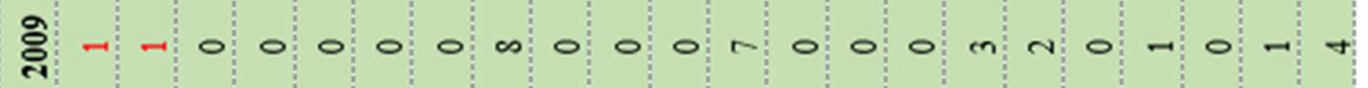
을 


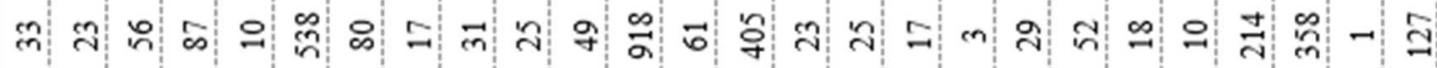

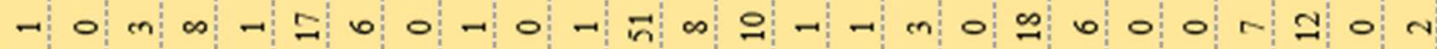
n 0000000000000000000000000 n $4 \infty m$ ก

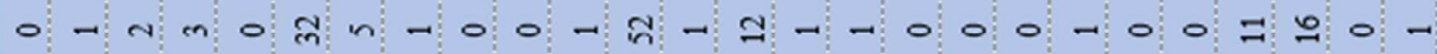
- m ๑ ○ 0 o 0 ○ - $\rightarrow$ -

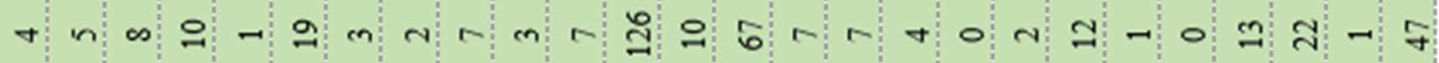
0000000000000000000000000 NoNJ

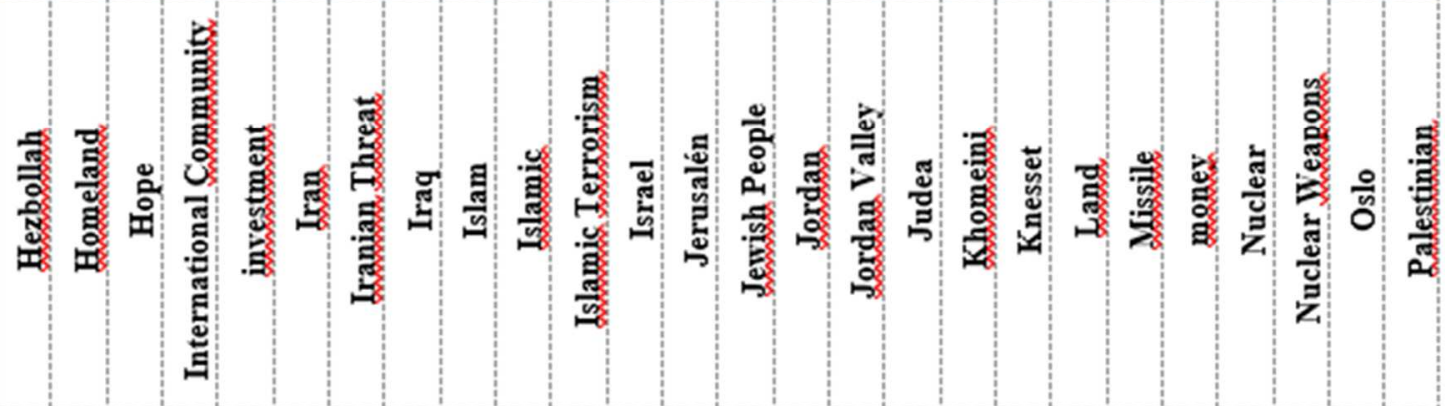


m NI

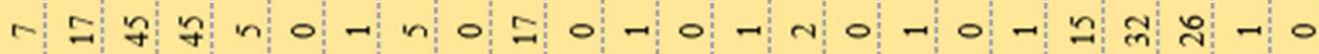
00000000000000000000000

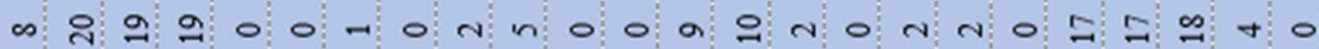

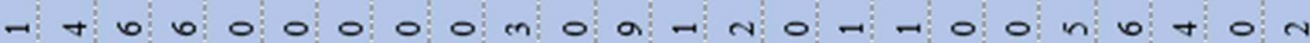

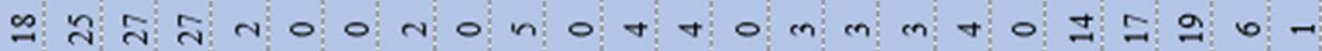
$a$ กัก๊ 이




П 00000000000000000000000

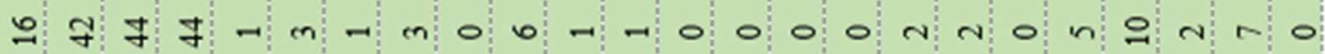

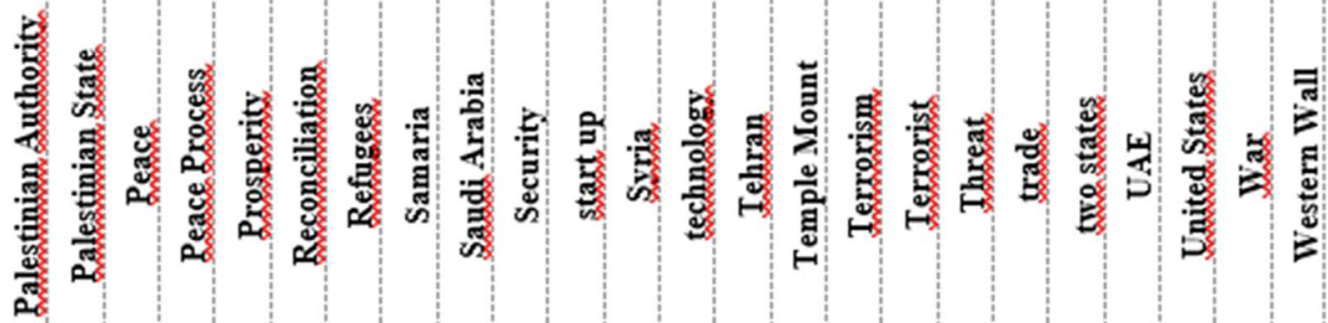




\section{Bibliografía}

\section{Fuentes Primarias}

Netanyahu, Benjamin (2009): “Israeli PM Netanyahu's Bar-Ilan Speech”, en https://ecf.org.il/media_items/1141.

Netanyahu, Benjamin (2011): "Speech by PM Netanyahu to the Joint Session of the US Congress" (22 mayo), en https://www.jewishvirtuallibrary.org/speech-by-pm-netanyahu-tojoint-session-of-the-u-s-congress-may-2011.

Netanyahu, Benjamin (2011): "Remarks by PM Benjamin Netanyahu to the U.N. General Assembly" (23 septiembre), en

https://mfa.gov.il/mfa/pressroom/2011/pages/remarks_pm_netanyahu_un_general\%20_assem bly_23-sep-2011.aspx.

Netanyahu, Benjamin (2012): "Prime Minister Benjamin Netanyahu's address to the United Nations General Assembly" (27 september), en https://www.haaretz.com/1.5182294 Fecha de consulta 21-6-2021.

Netanyahu, Benjamin (2012): "Netanyahu's speech at AIPAC" (6 marzo), en https://www.timesofisrael.com/netanyahus-speech-at-aipac-full-text/

Netanyahu, Benjamin (2013): "Netanyahu's 2013 speech to the UN General Assembly" (1 octubre), en https://www.timesofisrael.com/full-text-netanyahus-2013-speech-to-the-ungeneral-assembly/

Netanyahu, Benjamin (2013): "Speech at 2013 AIPAC National Policy Conference" (marzo),en https://www.jewishvirtuallibrary.org/prime-minister-netanyahu-speech-at-2013-aipacnational-policy-conference-march-2013 .

Netanyahu, Benjamin (2014): "Prime Minister Benjamin Netanyahu's Speech at the United Nations General Assembly” (29 septiembre), en https://www.likud.org.il/404

Netanyahu, Benjamin (2014): "Full transcript of Netanyahu's 2014 AIPAC address" (4 marzo), en https://www.timesofisrael.com/full-transcript-of-netanyahus-aipac-address/

Netanyahu, Benjamin (2015): "The complete transcript of Netanyahu's address to Congress" (3 marzo), en https://www.washingtonpost.com/news/post-politics/wp/2015/03/03/full-textnetanyahus-address-to-congress/

Netanyahu, Benjamin (2015): "Full text of Netanyahu 2015 address to the UN General Assembly" (1 octubre), en https://www.timesofisrael.com/full-text-of-netanyahu-2015address-to-the-un-general-assembly/

Netanyahu, Benjamin (2016): "Full text of Netanyahu's speech at 2016 UN General Assembly" (22 septiembre), en https://www.timesofisrael.com/netanyahus-full-remarks-at-un-generalassembly/

Netanyahu, Benjamin (2017): "Full text of Prime Minister Benjamin Netanyahu's UN speech" (19 septiembre), en https://www.timesofisrael.com/full-text-of-prime-minister-benjaminnetanyahus-un-speech/

Netanyahu, Benjamin (2018): “Address by H.E. Mr. Benjamin Netanyahu Prime Minister of Israel" (27 septiembre), en

https://gadebate.un.org/sites/default/files/gastatements/73/il_en.pdf 
Netanyahu, Benjamin (2018): "Benjamin Netanyahu addressed the 2018 AIPAC Policy Conference" (6 marzo), en https://www.jewishvirtuallibrary.org/benjamin-netanyahu-address2018-aipac

Netanyahu, Benjamin (2020): "Full text of Netanyahu's speech: Today recalls historic day of Israel's founding" (28 enero), en https://www.timesofisrael.com/full-text-of-netanyahusspeech-today-recalls-historic-day-of-israels-founding/

Netanyahu, Benjamin (2020): "Full text of Benjamin Netanyahu's address to the 2020 UN General Assembly" (29 septiembre), en https://www.timesofisrael.com/full-text-of-benjaminnetanyahus-address-to-the-2020-un-general-assembly/

Netanyahu, Benjamin (2021): "Full text: Netanyahu's furious final speech after 12 years as prime minister" (18 junio), en https://www.timesofisrael.com/full-text-netanyahus-furiousfinal-speech-after-12-years-as-prime-minister/

\section{Fuentes Secundarias}

Alvarez-Osorio, Ignacio "Para Israel la alternativa es la continuidad" Política Exterior, vol. 33, no192, (2019), pp. 27-32.

Arellano, Fernando "El «Mac Guffin» de Netanyahu. Consideraciones sobre el planteamiento israelí acerca del supuesto programa nuclear de Irán” IEEE, Nº 10, 2018, pp. 1139-1164.

Aron, Leon "The Putin Doctrine. Russia's Quest to Rebuild the Soviet State" Foreign Affairs, March 2013.

Atkins, Judi “A New Approach to Humanitarian Intervention? Tony Blair's "Doctrine of the International Community" British Politics, n¹(2) (February 2006), pp. 274-283

Avisai, Bernard "Netanyahu's Speech" New Yorker, (March 2015), en https://www.newyorker.com/news/news-desk/netanyahu-speech-congress

Bahar, Dany and Eckstein, Zvi "Israeli Voters Don't Care About the Economy. They Should" Foreign Policy, (September 2019)

Bahar, Dany (2016): "Delivering on economic prosperity in Israel. How monopolies are hampering the start-up nation", Brookings Institution, 4 May 2016, en https://www.brookings.edu/blog/markaz/2016/05/04/delivering-on-economic-prosperity-inisrael-how-monopolies-are-hampering-the-start-up-nation/

Bayme, Steven "Israel-diaspora relations in the age of Netanyahu" en Freedman, Robert O. (2019): Israel Under Netanyahu: Domestic Politics and Foreign Policy. Abingdon, Routledge.

Beinart, Peter: "How U.S. Jews Stymie Peace Talks", The Daily Beast, 27 de septiembre de 2010, en http://www.thedailybeast.com/articles/2010/09/27/us-jewish-groups-help-makepalestinian-state-impossible.html

Benn, Aluf: "The End of the Old Israel: How Netanyahu has transformed the nation" Foreign Affairs, vol, 96, nº 4, (2016) pp.16-27

Bostdorff, Denise (2008): Proclaiming the Truman Doctrine: The Cold War Call to Arms. Austin, Texas A \& M University Press

Broader, Jonathan "Netanyahu and the American Jew" World Policy Journal, (Spring 1998), pp. 89-98.

Caspit, Ben (2016): The Netanyahu Years, London, Thomas Dunne Books. 
Charney, Igal "A "Supertanker" Against Bureaucracy in the Wake of a Housing Crisis: Neoliberalizing Planning in Netanyahu's Israel", Antipode, vol. 49, nº 5 (2017), pp. 1223-1243

Dieckhoff, Alain "Israel: un cuarto mandato para Netanyahu" Afkar Ideas, n 59, (2019), pp. 43-45

Drake, Paula "Netanyahu Primer", Journal of Palestine Studies, 1996, vol. 26, n 1, (Autumn, 1996), pp. 58-69.

Elles, Diane "The Foreign Policy of the Thatcher Government" en Minogue, Kenneth and Biddiss, Michael (Eds) (1987): Thatcherism: Personality and Politics, Zurich, Springer.

Ephratt, Michal "The Silence Address: Silence as it Emerged from Media Commentators and Respondents, Following Prime Minister Netanyahu's 2015 Address at the UN" Israel Studies, $\mathrm{n}^{\circ} .3$ (22) (Fall 2017)

Espinosa, Ángeles "Israel inaugura en Emiratos Árabes su primera embajada en un país del Golfo" 21 de junio de 2021, El País, en https://elpais.com/internacional/2021-06-29/israelinaugura-en-emiratos-arabes-su-primera-embajada-en-un-pais-del-golfo.html

Espinosa, Ángeles "Irán dice que ha derribado un dron israelí cerca de una planta nuclear" El País, 24 de agosto de 2014, en https://elpais.com/internacional/2014/08/24/actualidad/1408890821_065821.html

Finkelstein, Justin S. "Netanyahu's Speech: What Difference Does it Make?" Foreign Policy Research Institute, (March 2015), en https://www.fpri.org/2015/03/netanyahus-speech-whatdifference-does-it-make/

Freedman, Robert O. (2019): Israel Under Netanyahu: Domestic Politics and Foreign Policy, Abingdon, Routledge.

Freedman, Robert O. "The erosion of US-Israeli relations during Obama's second term", Israel Affairs, vol. 23, n. 2, pp. 253-272.

García, David (2004) "Peace Through Primacy. La Administración Bush, La política exterior de EE.UU y las bases de una primacía Imperial. Geopolítica, recursos energéticos y Guerra al Terrorismo" UNISCI Discussion Papers, vol. 1, no 1 (enero 2004), pp. 1-31.

Glazer, Stephen “The Brezhnev Doctrine”, The International Lawyer, vol. 5, no.1, (January, 1971), pp. 169-179.

Gray, William (2003): Germany's Cold War: The Global Campaign to Isolate East Germany, 1949-1969, Chapel Hill, University of North Carolina Press.

Guardia de la, Julio "La profecía autocumplida de Netanyahu en Gaza" Política Exterior, vol. $28, n^{\circ} 161,(2014)$, pp. 24-30

Harel, Amos "Netanyahu, frente al cambio árabe y la amenaza iraní" Política Exterior, vol. 26, $\mathrm{n}^{\mathrm{o}} 145,2012$, pp. 22-30

Hoffman, Ronen (2019): Israel Foreign Policy under Benjamin Netanyahu, Foreign Policy Research Institute, Philadelphia.

Ilani, Ofri (2009): "Israel ranks fourth in the world in scientific activity, study finds". Haaretz, (17 de noviembre de 2009).

Kuttab, Daoud "Executive Overreach and Its Disastrous Results: The Case of Trump and Netanyahu" Palestine Israel Journal, vol. 24. nº. 3-4, (2017), pp. 175-180. 
Lagon, Mark (1994): The Reagan Doctrine: Sources of American Conduct in the Cold War's Last Chapter. Westport, Praeger.

Lochery, Neil (2016): The Resistible Rise of Benjamin Netanyahu. London, Bloomsbury.

Marcus, Jonathan "'Blast' deepens mystery of Iran's Parchin military complex" $B B C, 9$ de octubre de 2021, en https://www.bbc.com/news/world-middle-east-29550156

Morag, Shaqued "From Rabin's Peace to Netanyahu's Peace Conflict Management: The Ethos of and Israel's Younger Generation" Palestine Israel Journal, vol. 26, nº 1-2 (2019), pp. 42-49.

Nathason, Roby and Weiss, Yanai "The Israeli economy under the leadership of Netanyahu. Growth and stability at the cosrt of weakening the welfare state" en Freedman, Robert O. (2019): Israel Under Netanyahu: Domestic Politics and Foreign Policy. Abingdon, Routledge.

Navot, Doron and Rubin, Aviad: "Likud's success in the 2015 elections: Netanyahu's Hobbesian moment" Israel Affairs, vol. 22, nº 3-4, pp. 628-640.

Netanyahu, Benjamin (1997): Fighting Terrorism: How Democracies Can Defeat Domestic and International Terrorists, New York, Farrar, Straus and Giroux Publisher.

Netanyahu, Benjamin (2009): A Durable Peace: Israel and its Place Among the Nations, New York, Grand Central Publishing.

Ojeda de, Jaime "Carta de América: Obama-Netanyahu: pulso por el proceso de paz" Política Exterior, vol. 25, nº 142, 2011, págs. 8-13.

Ouimet, Matthew (2004) The Rise and Fall of the Brezhnev Doctrine in Soviet Foreign Policy. Chapel Hill, The University of North Carolina.

Pach, Chester: "The Reagan Doctrine: Principle, Pragmatism, and Policy" Presidential Studies Quarterly, vol 36, n 1 , (March 2006), pp. 75-88

Parsi, Trita (2007): Treacherous Alliance: The Secret Dealing of Israel, Iran and the United State, New Haven, Yale University Press

Pfeffer, Anshel (2017): Bibi: The Turbulent Life and Times of Benjamin Netanyahu. London, Basic Civitas Books.

Priego, Alberto (2021): "La inaplicabilidad de la Doctrina Begin en Irán: la Doctrina Bar Kojba" Documentos de Opinión IEEE, 70/2021, en http://www.ieee.es/Galerias/fichero/docs_opinion/2021/DIEEEO70_2021_ALBPRI_Iran.pdf

Priego, Alberto (2020): "El plan Trump: seguridad a cambio de bienestar" Documento de Opinión IEEE, 18/2020, en

http://www.ieee.es/Galerias/fichero/docs_opinion/2020/DIEEEO18_2020ALBPRI_planTrum p.pdf.

Priego, Alberto (2020): "La nueva política de Israel hacia el Golfo Pérsico" Documentos Opinión Instituto Español de Estudios Estratégicos, 147/2020, en http://www.ieee.es/Galerias/fichero/docs_opinion/2020/DIEEEO147_2020ALBPRI_Abraha m.pdf

Rumer, Eugene (2019) The Primakov (Not Gerasimov) Doctrine in Action. Washington D.C, The Carnegie Endowment for Peace.

del Sarto, Raffaella "Back to Square One? The Netanyahu Government and the Prospects for Middle East Peace", Mediterranean Politics, vol. 14, n'. 3 (November 2009), pp. 421-428.

Schilling, Christopher L. "The Ghetto Complex: Rethinking Israel's foreign policy”, The International Journal of Interdisciplinary Social Science, vol. 5, $\mathrm{n}^{\circ} .4$ (2010). 
Scott, James M. (1996): Deciding to Intervene: The Reagan Doctrine and American Foreign Policy. Durham, Duke University Press.

Senor, Dan and Singer, Saul (2009): Start-up Nation: The Story of Israel's Economic Miracle, New York, Twelve.

Snajder, Mario "Seguridad en Israel: un concepto flexible y cambiante" en Leal, Inma L., y Moloeznik, Marcos P. (Coord.) (2017): La política de defensa y seguridad de Israel como modelo, Guadalajara, Universidad de Guadalajara.

Schneider, Howard "Poll Gives Netanyahu Positive Marks Despite Rift with US". The Washington Post, 20 de marzo de 2009.

Stein, Aaron (2016): Turkey's New Foreign Policy: Davutoglu, the AKP and the Pursuit of Regional Order. Abingdon, Routledge.

Svirsky, Shlomo (2013): "Nes Gadol Haya Po. The economic policy of the second Netanyahu government, 2009-20122, Adva Center (November 2013), en

http://adva.org/wp-content/uploads/2015/01/\%D7\%A0\%D7\%A1-\%D7\%92\%

D7\%93\%D7\%95\%D7\%9C-\%D7\%9C\%D7\%90\%D7\%AA\%D7\%A81.pdf

Torres, José Ignacio "La propuesta de Trump para Palestina condiciones leoninas para un acuerdo imposible" IEEE, no 17, 2020, pp. 19-28.

Ziv, Guy: "Benjamin Netanyahu's Calculated Ambiguity Toward the Two-State Solution", Political Science Quarterly, nº 2 (13) (2018-19) DOI: 10.1002/polq.12900 2 